\title{
Center for Research on Economic and Social Theory
}

and

Department of Economics

Working Paper Series

Simulated Maximum Likelihood Estimation of Dynamic Discrete Choice Statistical Models---Some Monte Carlo Results

Lung-fei Lee

April 1994

Number 94-06

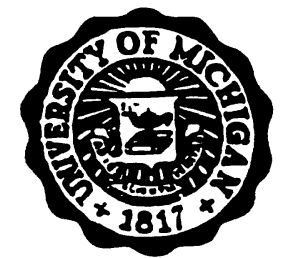

DEPARTMENT OF ECONOMICS

University of Michigan

Ann Arbor, MI 48109-1220
SUMNER AND LAURA

FOSTER LIBRARY

NUL I 3 Bon 


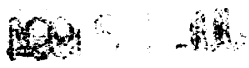


Simulated Maximum Likelihood Estimation of Dynamic Discrete Choice Statistical Models - Some Monte Carlo Results

\section{by Lung-Fei Lee}

Department of Economics, The University of Michigan

Ann Arbor, MI 48109-1220

April 1994

\section{Abstract}

This article reports Monte Carlo results on the simulated maximum likelihood eatimation of discrete panel atatistical models. Among them are Markov, Generalized Poly, Renewal, and Habit Persistence Modelo with or without unobserved heterogeneity and serially correlated disturbances. We investigate statistical properties and computational performance of simulated maximum likelihood methods and a bias-correction procedure. With a moderate number of simulation draws for the construction of simulator and the bian adjuated procedure, moot of these complex dynamic models can be adequately eatimated for panele with length up to 30. The Polya model and the Renewal model can be accurately eatimated for panelo with 50 periods.

\section{JEL subject codes: C13, C15, C23, C25}

\section{Key Worda:}

Discrete choice panel models, dynamic models, heterogeneity, serial correlation, simulated ML, biascorrection, Monte Carlo studiea.

CORRESPONDENCE ADDRESS:

Lung-Fei Lee, Department of Economics, The Univeroity of Michigan, 611 Tapan Street, Ann Arbor, MI 48109-1220.
Simulated Maximum Likelihood Eatimation of Dynamic Discrete Choice Statistical Models - Some Monte Carlo Reaults

by Lung-Fei Lee*

1. Introduction

In Heckman (1981a), a rich group of discrete time-discrete outcome stochastic proceseses has been introduced. It contains Bernoulli models, Markov models, renewal procesees, Polya models, latent Markov models, and other familiar stochastic proceseses. The models are sufficiently flexible to accommodate timo-varying explanatory variables, serial correlation for unobeervable, and complex structural economic interrelationships among decisions over time. With rich specifications on both lagged dependent variables and correlated disturbances, these proposed models accommodate true and spurious state dependence and can be used to test competing theories on state dependence. Heckman (1981a) has provided discussions on various models in terms of data requirements, model identification, and implications for probability run patterns. Implomentation of probability run patterns to test true state dependence sgainst spurious state dependence can also be found in Heckman (1978) and Lee (1987).

For computation, Heckman (1981a) has focused on models with fixed effect, random components, or factor analytic schemes for unobserved heterogeneity but with serially independent distribution for the remaining disturbance. Such structures simplify computation as likelihood functions for ouch modelo involve single integral, which can be effectively implemented by the Gauevian Quadrature method. While the specification of random component for unobserved heterogeneity is a popular approach for panel data, the serially independent assumption for the remaining disturbance is restrictive. Likelihood functions for models with general serial correlation will in general involve multiple integrale. For diecrete panel dats modele, the dimension of multiple integrals will be proportional to the number of time periode. The numerical implementation of such models is known to be computationally difficulty if not intractable. However, the recent development of simulation methods by Lerman and Manoki (1981), McFadden (1989), Pakes and Pollard (1989), Borsch-Supan and Hajivaseiliou (1993), and Keane (1994), among others, provides evidences that such a difficulty may be overcome with carefully designed Monte Carlo simulation eatimation methoda. $\mathbf{A}$ simulator due to Geweke (1989), Borech-Supan and Hajivaseiliou (1993), and Keane (1994) bacod on recuraive simulation of truncated random variables is chown to provide a good unbiasod aimulator for multivariate * I appreciate having financial support from the National Science Foundation under grant number SBR9223325 for my research. 
normal probabilities. Keane (1994) has emphasized its usefulness for the estimation of panel data model and has provided some Monte Carlo results for binary choice panel regression models with 8 periods. The Model Carlo results seem encouraging.

In this article, we are going to investigate the simulated maximum likelihood method for the estimation of various dynamic panel models introduced in Heckman (1981a). Special attention will be on the value and potential limitation for the estimation of long panels. We report Monte Carlo results for the simulation estimation of such models. An important aspect of the experiment is to investigate the discrimination ability of the true state dependence vs. spurious state dependence in various dynamic models. Other aspects of interest are on the simulated likelihood method itself. As the time length for a panel data increases, the total number of choice alternatives, which are composed of choice paths, increases exponentially and the dimension of integration increasea linearly. The probability for any specific choice path will become smaller for longer panel data. One may expect that the simulator would become less accurate as time length increases. So one of the aspects is to investigate the quality of the simulated estimates for model parameters with long panel data. As the likelihood function is nonlinear, the simulated log likelihood function is a biased estimate of the exact log likelihood function. The simulated likelihood estimator can be consistent only if the number of simulated variables in the construction of simulator increases with sample size. For efficient estimation, the number of simulated variables has to increase with sample size at a certain rate. If the number of simulated variables does not increase fast enough or, in practice, the number is not large enough relative to sample size, the simulated likelihood estimator might have an asymptotic bias or a dominated finite sample bias (relative to standard error) due to simulation. The leading bias term due to simulation in the simulated likelihood estimator can be removed by a bias-correction procedure as auggested in Lee (1993). As a by-product, we will investigate value of the bias-correction procedure for the estimation of dynamic models with our Monte Carlo experiments.

The organization of this article is as follows. In Section 2, we provide an overview of various interesting models introduced in Heckman (1981a). It provides a guided tour for models studied in our Monte Carlo experiments. We review the formulation of simulators and simulated likelihood functions for such modele and describe the bias-correction procedure. Monte Carlo results are reported for each model in subsequent sections. General conclusions are drawn in the last section.
2. Dynamic Models and Simulated Maximum Likelihood Estimation - an Overview

To analyze panel discrete responses in discrete time, Heckman (1981a) proposed a general framework in terms of latent dependent variables. Reeponses are observed from signs of latent dependent variables. The latent dependent variable at each period can be interpreted as utility difference scroos choice alternatives. Observed responses are results of utility maximization as in McFadden's discrete choice framework (1974). The latent dependent variable $y_{i t}^{*}$ for an individual $i$ at period $t$ is a function of vector of exogenous variablen $z_{i i}$, past occurrence of the event, and prior propensities to select a state:

$$
y_{i t}^{*}=z_{i t} \beta+\sum_{i=1}^{\infty} \gamma_{l} y_{i, t-1}+\lambda \sum_{i=1}^{\infty} \prod_{l=1}^{\infty} y_{i, t-1}+\sum_{i=1}^{\infty} \delta_{l} y_{i, t-1}^{*}+v_{i t,}
$$

and the observed dependent variable is

$$
y_{i t}= \begin{cases}1 & \text { if } y_{i t}^{*}>0, \\ 0 & \text { if } y_{i t}^{+} \leq 0 .\end{cases}
$$

The initial conditions $y_{i t}$ and $y_{i t}^{*}, t=0,-1, \ldots$, are assumed to be fixed outside the model. The vector of disturbances $v_{i}=\left(v_{i 1}, \cdots, v_{i T}\right)^{\prime}$ are independent of all $z_{i t}$ and are independent acroes individuals, but its components can be serially correlated over time.

The first term on the right-hand side of (2.1) represents the effect of exogenous variables on choice. The second term on the right-hand side of (2.1) represents the effect of the entire past history of the procese on current choice. The third term represents the cumulative effect of the continuous experience in a atate on current choice. This term could be generalized to allow for depreciation. The fourth term captures the notion of habit persistence. This general model can further be generalized to allow coefficients to depend on time as indicated in Heckman (1981a). This specification accommodates a wide variety of interesting atochastic models. The model that $y_{i t}=z_{i t} \beta+\sum_{t=1}^{\infty} \gamma_{i} y_{i, t-1}+v_{i t}$ includes the Markov proceses and the Polya model. The specification $y_{i t}^{i}=z_{i t} \beta+\lambda \sum_{s=1}^{\infty} \prod_{i=1}^{i} y_{i, 1-1}+v_{i t}$ generates a renewal proceso. A latent Markov model emerges in $y_{i t}^{*}=z_{i t} \beta+\sum_{i=1}^{\infty} \delta_{i} y_{i, t-1}^{*}+v_{i t}$. Our Monte Carlo experiment will otudy these various interesting models.

An important issue for panel models is to distinguish true state dependence from spurious atate dopendence. In true state dependence, past experience has a genuine behavioral effect in that an identical individual who has not experienced the event will behave differently than an individual who has experienced the event. The introduction of lagsed variables of $y$ or $y^{*}$ captures such a dependence. In apurious state dependence, previous experience appears to influence the determinant of future event eolely because it in 
due to temporally correlated and persistent unobservable that determine choices. The unobservable heterogeneity for spurious state dependence can be introduced into the model with serially correlated disturbances and a random individual component in $v_{i t}$ for example, $v_{i t}=\xi_{i}+\epsilon_{i t}$, where $\epsilon_{i t}$ can be serially correlated and $\xi_{i}$ is an individual component. With ouch a specification, the general framework (2.1) accommodates general sorts of heterogeneity and state dependence. To distinguish true state dependence from spurious state dependence, an alternative simple method based on lagged exogenous variables has been suggested in Chamberlain (1978). However, in contrary to the framework (2.1), that simple method can not make distinctions with regard to different types of state dependence, heterogeneity, and serial correlation. Hsiao (1986) provides a comprehensive survey on these models and related issues.

For the eatimation of (2.1), Heckman (1981a) has proposed the method of maximum likelihood under the assumption that $v_{i 1}, \ldots, v_{i r}$ are multivariate normally distributed. A general likelihood function can be found in his article.' For general correlated disturbances, the likelihood function involves multiple integralo. In a limited Monte Carlo experiment reported in Heckman (1981b) and an empirical application in Heckman (1981c), only fixed effect and random component models with $v_{i t}=\xi_{i}+\epsilon_{i t}$, where $\epsilon_{i t}$ are serially uncorrelated, are estimated. For random component or one factor models, multivariate probability functions involve only single integrals, which can be effectively implemented by the Gauseian Quadrature method [Butler and Moffitt (1982)]. For more general correlated disturbances, we suggest estimation by simulated maximum likelihood methods. Simulation based estimation methods have recently been proposed by Lerman and Manski (1981), McFadden (1987), and Pakes and Pollard (1987), among others. For the implementation of simulated likelihood methods, we adopt the Geweke-Hajivassiliou-Keane (GHK) simulator. The GHK simulator is an unbiased simulator that can provide close approximation to some multivariate normal probabilities (Borsch-Supan and Hajivassiliou, 1993) and is of special interest for panel data models (Keane, 1994).

To illuatrate the construction of the GHK simulator and the simulated likelihood function for (2.1) that are relevant for our experiment, consider the opecification $v_{i t}=\sigma \xi_{i}+c_{i t}$, where $c_{i t}$ is a $\operatorname{ARMA}(p, q)$ process: $c_{i t}=\sum_{i=1}^{p} \rho_{1} \epsilon_{i, t-1}+w_{i t}+\sum_{j=1}^{\prime} \psi_{j} w_{i, t-j}$. The initial values for $c_{i t}, w_{i t}, \psi_{i t}$ and $y_{i t}^{i}$ for $t \leq 0$ are known and are asoumed to be zero. For normalization, $w_{i t}$ has $a$ unit variance. Also $\xi$ has a unit variance in the above specification. To simplify notation, the individual subecript $i$ will be dropped in the derivation of the ${ }^{1}$ The likelihood formula there is correct only for models without lagged latent dependent varisbles. His likelihood formula needo to be revised for models with lagged latent dependent variables. likelihood function for an individual. Let $a_{t}=z_{t} \beta+\sum_{l=1}^{\infty} n y_{t-1}+\lambda \sum_{t=1}^{\infty} \prod_{i=1}^{i} y_{t-1}+\sum_{l=1}^{\infty} \delta_{1} y_{i-1}^{i}+\sigma \xi$. The joint probability for $y_{1}, \ldots, y_{r}$ conditional on exogenous variables and $\xi$ is

$$
P\left(y_{1}, \ldots, y_{T} \mid z_{1}, \ldots, z_{T}, \xi\right)=\int_{L_{1}}^{U_{1}} \cdots \int_{L_{T}}^{U_{T}} f\left(\epsilon_{T} \mid \epsilon_{T-1}, \cdots, \epsilon_{1}\right) f\left(\epsilon_{T-1} \mid \epsilon_{T-2}, \cdots, \epsilon_{1}\right) \ldots f\left(\epsilon_{1}\right) d \epsilon_{T} \ldots d \epsilon_{1}, \text { (2.2) }
$$

where $f\left(\epsilon_{1} \mid \epsilon_{t-1}, \cdots, \epsilon_{1}\right)$ is the conditional density of $\epsilon_{t}$ conditional on the past $\epsilon_{s}$ and the integral limits are

$$
L_{t}=\left\{\begin{array}{ll}
-a_{t} & \text { if } y_{t}=1, \\
-\infty & \text { if } y_{t}=0,
\end{array} \quad U_{t}= \begin{cases}\infty & \text { if } y_{t}=1, \\
-a_{t} & \text { if } y_{t}=0 .\end{cases}\right.
$$

Given the initial conditions for $e$ and $w$, the ARMA proceses can be inverted recuraively wo that each $w_{\boldsymbol{t}}$ can be expressed as a function of $c_{t-o}, s \geq 0$. Denote $b_{t}=a_{t}+\sum_{i=1}^{p} \rho_{1} \epsilon_{t-1}+\sum_{j=1}^{\prime} \psi_{j} w_{t-j}$. Let $\phi$ and $\$$ be, respectively, the standard normal density and distribution functions. Extend the limits $L_{\mathbf{t}}$ and $U_{1}$ to

$$
\bar{L}_{t}=\left\{\begin{array}{ll}
-b_{t} & \text { if } y_{t}=1, \\
-\infty & \text { if } y_{t}=0,
\end{array} \quad \bar{U}_{t}= \begin{cases}\infty & \text { if } y_{t}=1, \\
-b_{t} & \text { if } y_{t}=0 .\end{cases}\right.
$$

By a transformation of variables, (2.2) becomes

$$
\begin{aligned}
& P\left(y_{1}, \ldots, y_{r} \mid z_{1}, \ldots, z_{T}, \xi\right) \\
& =\int_{L_{1}}^{O_{1}} \cdots\left(\int_{L_{T-1}}^{O_{T-1}}\left[\int_{L_{T}}^{O_{T}} \phi\left(w_{T}\right) d w_{T}\right] \phi\left(w_{T-1}\right) d w_{T-1}\right) \cdots \phi\left(w_{1}\right) d w_{1} \\
& =\int_{-\infty}^{\infty} \ldots \int_{-\infty}^{\infty}\left(\Phi\left(\bar{U}_{T}\right)-\Phi\left(\bar{L}_{T}\right)\right) \prod_{i=1}^{T-1}\left(\Phi\left(\bar{U}_{T-\jmath}\right)-\Phi\left(\bar{L}_{T-\jmath}\right)\right) \phi_{\left(L_{T-\ldots} .0_{T-\jmath}\right)}\left(w_{T-\lrcorner}\right) d w_{T-\lrcorner} \\
& =\int_{-\infty}^{\infty} \cdots \int_{-\infty}^{\infty} \Phi\left(\left(2 y_{T}-1\right) b_{T}\right) \prod_{t=1}^{T-1} \Phi\left(\left(2 y_{T-0}-1\right) b_{T-0}\right) \phi_{\left(z_{T-0}, 0_{T-0}\right)}\left(w_{T-0}\right) d w_{T-\infty},
\end{aligned}
$$

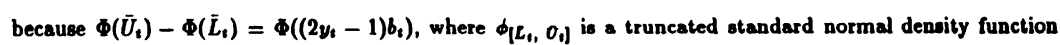
with support $\left[\bar{L}_{t}, \bar{U}_{t}\right]$. The probability of $y_{1}, \cdots, y_{T}$ conditional on $z_{1}, \cdots, z_{T}$ is $P\left(y_{1}, \cdots, y_{T} \mid z_{1}, \cdots, z_{T}\right)=$ $\int_{-\infty}^{\infty} P\left(y_{1}, \cdots, y_{T} \mid z_{1}, \cdots, z_{T}, \xi\right) \phi(\xi) d \xi$. This function and $(2.3)$ naturally ouggest an unbiased cotimator, namely, the GHK aimulator.

The truncated atandard normal variable $w_{t}$ on $\left[\bar{L}_{t}, \bar{U}_{t}\right]$ can be easily simulated a traneformation of a uniform random variable. Suppose $u$ is a random draw from the uniform $[0,1]$ distribution. Corresponding to $y_{t}=0, \bar{L}_{t}=-\infty$ and $\bar{U}_{t}=-b_{t}$. A truncated atandard normal variable $w_{t}$ can be obtained from the relation $\Phi\left(w_{t}\right) / \Phi\left(-b_{t}\right)=u$. When $y_{t}=1, \bar{L}_{t}=-b_{t}$ and $\tilde{U}_{t}=\infty$. For the latter case, the truncated atandard normal variable can be obtained from $\left[\Phi\left(w_{t}\right)-\Phi\left(-b_{t}\right)\right] /\left[1-\Phi\left(-b_{t}\right)\right]=1-u$. Using the aymmetric property of the atandard normal density, $w_{t}=-\left(2 y_{t}-1\right) \Phi^{-1}\left[u \Phi\left(\left(2 y_{t}-1\right) b_{t}\right)\right]$ is a random draw from the truncated otandard normal distribution on $\left[\bar{L}_{t}, \bar{U}_{t}\right]$. The random variables for the GBK simulator can be recuraively 
generated. Initiate $\epsilon_{0}=0$ and other initial conditions. Generate $u_{1}, \cdots, u_{T-1}$ independent uniform $[0,1]$ random variables. Generate $\xi$ from a $N(0,1)$ random variable generator. The random variables $w_{1}, \cdots, w_{T-1}$ can then be generated recursively from the following steps from $t=1$ to $T-1$ :

1). Compute

$$
\begin{gathered}
w_{t}=-\left(2 y_{t}-1\right) \Phi^{-1}\left[u _ { t } \Phi \left(( 2 y _ { t } - 1 ) \left(z_{t} \beta+\sum_{i=1}^{\dot{1}} \gamma_{l} y_{t-1}+\lambda \sum_{j=1}^{1} \prod_{l=1}^{\infty} y_{t-1}+\sum_{i=1}^{1} \delta_{l} y_{i-1}^{*}\right.\right.\right. \\
\left.\left.\left.+\sigma \xi+\sum_{i=1}^{p} \rho_{i} \epsilon_{t-1}+\sum_{j=1}^{\dot{1}} \psi_{j} w_{t-j}\right)\right)\right] .
\end{gathered}
$$

2). Update the disturbance process $c_{i}=\sum_{i=1}^{p} \rho_{1} \epsilon_{t-1}+w_{i}+\sum_{j=1}^{p} \psi_{j} w_{t-j}$.

3). Generate the latent dependent variable

$$
y_{i}^{*}=z_{t} \beta+\sum_{i=1}^{i} \gamma_{l} y_{t-1}+\lambda \sum_{i=1}^{1} \prod_{l=1}^{\prime} y_{t-1}+\sum_{i=1}^{\dot{1}} \delta_{l} y_{i-1}+\sigma \xi+\epsilon_{t}
$$

For special models, some of the above simulation steps may not be needed. For example, if the model does not involve lagged latent dependent variable $y^{*}$, simulation of the latent variables $y_{i}^{*}$ will not be needed and the step 3) can be pased over. For each $i$, with $m$ independently generated vector of random draws, the simulated log likelihood function is

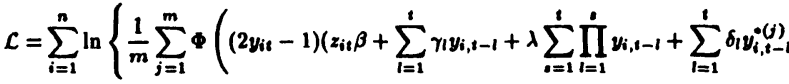

$$
\begin{aligned}
& \left.\left.\left.+\sigma \xi_{i}^{(j)}+\sum_{i=1}^{p} \rho_{1} \epsilon_{i, 1-1}^{(j)}+\sum_{j=1}^{\infty} \psi_{j} w_{i, t-j}^{(j)}\right)\right)\right\}
\end{aligned}
$$

where the superscript $(j)$ denotes an independent simulation draw.

Asymptotic properties of the simulated maximum likelihood estimator (SMLE) have been studied in Hajivassiliou and McFadden (1990), Lee (1992, 1993) and Gourieroux and Monfort (1993), among others. As the choice probability simulatore are independent across $i$, the simulated MLE can be asymptotically efficient when $\lim _{n \rightarrow \infty} n^{1 / 2} / m=0$. However, when $m$ increases at a rate slower than $n^{1 / 2}$, the limiting distribution of the SMLE becomes nasty. As shown in Lee (1993), if $m$ increases at the rate of $n^{1 / 2}$, an asymptotic bias existo in the limiting distribution. The situation becomes worse when $m$ increases at a rate slower than $n^{\mathbf{1} / 2}$ because the asymptotic bias will then dominate the variance. To reduce the dominated bias due to simulation, Lee (1993) has suggested a simple bias-correction procedure. For the discrete choice model with $L$ alternatives, suppose the simulated log likelihood is written in a familiar format as $\mathcal{L}(\theta)=\sum_{i=1}^{n} \sum_{i=1}^{L} d_{i j} \ln f_{m, l, i}\left(z_{i}, \theta\right)$ where $\theta$ is the vector of parameters in the model, $d_{1}$ is the dichotomous choice indicator for the alternative $I$ and $f_{m, l, i}=\frac{1}{m} \sum_{j=1}^{m} h\left(w_{i}^{(j)}, z_{i}, \theta\right)$ is the simulated probability for the alternative $l$ for the individual $i$. Let $\hat{\theta}$ be the SMLE. A bias-adjusted estimator $\hat{\theta}_{A}$ can be derived by correcting a bias term from $\hat{\theta}$ :

$$
\hat{\theta}_{A}=\hat{\theta}-\left\{\sum_{i=1}^{n} \sum_{i=1}^{L} d_{i j} \frac{\partial \ln f_{m, l, i}\left(z_{i}, \hat{\theta}\right)}{\partial \theta} \frac{\partial \ln f_{m, l, i}\left(z_{i}, \hat{\theta}\right)}{\partial \theta^{\theta}}\right\}^{-1} \sum_{i=1}^{n} \omega_{m, i}\left(z_{i}, \hat{\theta}\right) / m,
$$

where

$$
\begin{gathered}
\omega_{m, i}\left(z_{i}, \theta\right)=\sum_{i=1}^{L}\left\{\frac { d _ { i l } } { f _ { m , l , i } ^ { 2 } ( z _ { i } , \theta ) } \left[\frac{\partial \ln f_{m, l, i}\left(z_{i}, \theta\right)}{\partial \theta}\left(S_{m, l, i}\left(z_{i}, \theta\right)-f_{m, l, i}^{2}\left(z_{i}, \theta\right)\right)\right.\right. \\
\left.\left.-\left(C_{m, l, i}\left(z_{i}, \theta\right)-f_{m, l, i}\left(z_{i}, \theta\right) \frac{\partial f_{m, l, i}\left(z_{i}, \theta\right)}{\partial \theta}\right)\right]\right\},
\end{gathered}
$$

with $S_{m, l, i}\left(z_{i}, \theta\right)=\frac{1}{m} \sum_{j=1}^{m} h_{l}^{2}\left(w_{i}^{(j)}, z_{i}, \theta\right)$ and $C_{m, l, i}\left(z_{i}, \theta\right)=\frac{1}{m} \sum_{j=1}^{m} h_{l}\left(w_{i}^{(j)}, z_{i}, \theta\right) \frac{\theta h_{1}\left(\varphi_{j}^{\left.(j), x_{i}, \theta\right)}\right.}{\partial j}$. The biescorrected estimator $\hat{\theta}_{A}$ improves upon $\hat{\theta}$ by eliminating the leading bias term in $\hat{\theta}$ due to simulation. Another valuable property of $\hat{\theta}_{A}$ is that its asymptotic efficiency requires only that $m$ goes to infinity at a rate faster than $n^{1 / 4}$ instead of $n^{1 / 2}$

The following sections provide Monte Carlo results for several interesting panel regression and dynamic choice models. We are interested on the performance of simulated maximum likelihood methods; namely, finite sample statistical properties of the SMLE and bias-corrected eatimators, the value of long panel data, and the time cost for estimation of various models. Some limited Monte Carlo results on the GHK simulator can be found in Borsch-Supan and Hajivaseiliou (1993) for multiple choice modela, and Keane (1994) for panel choice regression models up to eight time periods. Bias-corrected procedures have not been considered in their articles but in Lee (1993) for panel choice regression models and Markov models with four periods. Our present Monte Carlo experiments provide much more evidences on the value of simulated likelihood eatimation. Our experiments investigate both the aimulated maximum likelihood method and the biercorrection procedure. Panel models with periods much longer than eight are eatimated. We are interested in the estimation of various dynamic models in addition to regression modela. Practical values of simulation estimation are the ultimate goal of simulation based inference. The following Section 3 investigates only discrete choice regression models. Section 4 investigates Markov models. Polys and Renewal dynamic modelo are studied, respectively, in Sections 5 and 6. Section 7 considers Habit Persistent models. For all these models, first order autocorrelated disturbances are maintained. For models with unobeerved heterogeneity, additional random components are included. 


\section{Panel Discrete Choice Regression Modele}

3.1 Models with Serially Correlated Disturbancen

The discrete choice regression models are models without true state dependence. Throughout all the experiments, the stochastic process for the disturbance $c_{i t}$ is assumed to be a AR(1) process. The model for generating the sample for our experimental atudy is a binary choice panel model:

$$
y_{i t}^{*}=\lambda+\beta x_{i t}+\epsilon_{i t}
$$

and

$$
\epsilon_{i t}=\rho \epsilon_{i, t-1}+w_{i t},
$$

where $w_{i t}$ are i.i.d. normal $N(0,1)$. The vector of exogenous variables $z_{i t}$ is $\left(1, x_{i t}\right)$.

The exogenous variables $x_{i t}$ are generated as $x_{i t}=(1 / \sqrt{2}) r_{i t}+\sqrt{6} s_{i}$ where $r_{i t}$ are i.i.d. truncated normal $N(0,1)$ variables with support $[-2,2]$ and $s_{i}$ are independent uniform variables with support on $[-1 / 2,1 / 2]$. The variance of $\boldsymbol{x}$ is about 1 and its correlation coefficient over time is about 0.5 . This process of generating exogenous variables is used for all the models in this article. The initial condition for (3.2) is $\boldsymbol{c}_{i 0}=0$ for all i. Sample data are generated with $\beta=1, \lambda=0.2$ and $\rho=0.4$. This $\rho$ represents a moderate amount of sutocorrelation for the disturbance. The sample size is 200 . We have experimented with a small number of random draws and a moderate number of draws, namely, $m=15$ and $m=50$, for the construction of the GHK simulator. The simulated log likelihood function for this model is

$$
\mathcal{L}=\sum_{i=1}^{n} \ln \left\{\frac{1}{m} \sum_{j=1}^{m} \prod_{t=1}^{T} \Phi\left[D_{i t}\left(\lambda+\beta x_{i t}+\rho \epsilon_{i, t-1}^{(j)}\right)\right]\right\} .
$$

The number of periods for the panel data varies from $T=8$ to the maximum of 100 . For each case, the number of replications is 300. For each replication, the set of exogenous variables is redrawn. We report the empirical mean and standard deviation of estimates for each parameter. For the sake of easy comparison, we will aloo report root mean square errors for estimates and the CPU time in seconds per replication. All the computation is performed with IBM RS/6000 machines. The CPU time refers to the computation speed of - IBM RS/6000 Model 580 workstation. ${ }^{2}$ The maximization algorithm used is a conjugate gradient method described in Press et al. (1986), Chapter 10. For all the cases and replications reported here, the algorithm 2 Comparing the speed of Model 580 with the model $320 \mathrm{H}$ machine, the latter is slow by a time factor of 2.8 converges without running into numerical problems.3

Table 1.A reports empirical means and atandard deviations for both the SMLE (Bias unadjusted SML) and the bias-adjusted estimates (Bias adjusted SML). Root mean squared errors of eatimates are reported in Table 1.B. For all the panels with periods from 8 to 100, the SMLE of the intercept $\lambda$ and the regreasion slope parameter $\beta$ have small biases. The SMLE of the autocorrelation coefficient $\rho$ has shown some biases. As the number of time periods increases, the choice pattern becomes numerous and the choice probabilities become small. As smaller probabilities are more difficult to simulate than larger probabilities (Borsch-Supan and Hajivasailiou (1993)), biasea of the SMLE for long panels become alightly larger. On the other hand, since longer panel data provide more sample information about the stochastic procese, otandard errore of estimates decrease. Root mean square errors also decrease for estimates with longer panels if time periods are not too long. However, for long panels with $T=50$ or $T=100$, root mean square errors for $\rho$ are larger. For $T=100$, root mean square errors for $\beta$ are also larger. This is due to the circumstance that increasing bias may dominate the reduction of standard error. Biases of eatimates are all reduced when the number of simulated random variables increases from $m=15$ to 50 . The improvement of the eatimate of $\rho$ is more apparent. The increased CPU time coot is close to be linear in $m$ and $T$. The bias-corrected procedure eliminates some of the biases. The bias correction is valuable especially for small $m$ with $m=15$. Although standard errors of bias-corrected estimates are slightly larger, the reduction in bias dominates the slight increase of standard error and results in smaller root mean squares errors for estimates of $\rho$. Root mean square errors of bias-corrected estimates are in general smaller than root mean square errors of bias. unadjusted estimates for long panels. For short panels with $T=8$ and 15 , bias-corrected estimates with $m=15$ can be at least as good as unadjusted SMLE with $m=50$ in terms of similar bias. The additional CPU cost for bis-correction is negligible in the overall cost. In sum, the performance of the simulated ML methods seems satisfactory ever for long panels in this experiment.

Tables 2.A and 2.B report results for another data set. The sample is generated with $\rho$ set to 0.85 , a rather high correlation for the disturbance. The SMLE have much larger biaces when they are compared with corresponding estimates in the previous sample with $\rho=0.4$. Estimates with shorter periods $T=8$ and 15 seem satisfactory even with $m=15$. But for panels with longer periods, biases of estimates seem 3 For models without unoboerved heterogeneity, initial catimates are arbitrarily set to zero. For models with unobservable heterogeneity, the initial estimate of $\sigma$ is set to one and the initial coltimales of the othe $(\beta, \lambda, \rho)$ with which the algorithm converges to similar solutione. 
severely downward. The results are better for the biased-corrected procedure. But for better improvement, larger $m$ is desirable. With $m=50$, the SMLE are reasonably good even for $T=50$. For strong serial correlation, estimates for $\rho$ have smaller standard errors but estimates of the intercept and the regression coefficient have larger errors than corresponding cases with a moderate serial correlation. With atrong serial correlation, one may expect that there would be less variation and less changes on choices over time. Overall, simulation estimates perform better for models with moderate serial correlation than models with rather high serial correlation. The Monte Carlo results in Keane (1994) for a similar model with additional unobserved heterogeneity pointed out the same problem.

3.2 Models with Unobserved Heterogeneous and Serially Correlated Disturbances

The model with unobserved heterogeneity generalizes the previous model (3.1)-(3.2) by introducing a random component as an additional disturbance:

$$
y_{i t}^{:}=\lambda+\beta x_{i t}+\sigma \xi_{i}+\epsilon_{i t},
$$

where $\xi_{i}$ are i.i.d. $N(0,1)$ and are independent of $\epsilon_{i s}$. The simulated log likelihood function for this model is

$$
\mathcal{L}=\sum_{i=1}^{n} \ln \left\{\frac{1}{m} \sum_{j=1}^{m} \prod_{t=1}^{T} \Phi\left[D_{i t}\left(\lambda+\beta x_{i t}+\sigma \xi_{i}^{(j)}+\rho \epsilon_{i, t-1}^{(j)}\right)\right]\right\} .
$$

Sample data are generated with parameters $\lambda=0.2, \beta=1.0, \sigma^{2}=0.5$, and $\rho=0.4$. With the additional $\sigma \xi$, the serial correlation of two adjacent periods has a correlation coefficient about 0.6 and the fraction of variance due to the individual effect is about 0.3 .

Tables 3.A and 3.B report simulated likelihood estimates and their root mean squares errors. All the estimates for $\beta$ have small biases. Biases for the intercept $\lambda$ are also small for $T$ up to 50 . There are some biases for the SMLE of $\sigma$ and $\rho$. The SMLE of $\sigma$ tend to increase as $T$ increases and are biased upward for long panels with $T=50$ or 100 . On the other hand, biases of $\rho$ are downward. So for long panel data, the correlation due to unobserved heterogeneity tends to be over estimated and the serial correlation of the remaining disturbance tends to be underestimated. ${ }^{5}$ With $m$ increases to 50 , biases are reduced and the SMLE are reasonably good for $T$ up to 30 . The bias correction procedure reduces some of the biases and root mean square errors in $\beta$ and $\rho$. Standard errors for estimates of $\beta$ and $\rho$ are decreasing as periodo in 1 As Keane (1994) has experimented with a very amall $m$, namely 10 draws, and has neither used any bias correction procedure nor increased the number of simulation draws, his conclusion is too pessimistic. en in in hane (1994) bas a deo but maller fraction of variance due to the individual eftect, the Monte Carlo panels increase. For long panels with $\boldsymbol{T}=\mathbf{5 0}$ and 100, biases can dominate standard errors that results in larger root mean square errors. Overall, thooe SMLE and bias-corrected estimates with $m=50$ are good for panels with $T$ up to 30 for this model. Comparing these estimates with estimates of panel regreseion models in (3.1) without unobserved heterogeneity, these estimates are slightly worse than the corresponding estimates in Table 1 with $\rho=0.4$ but are much better than the estimates in Table 2 with $\rho=0.85$. This may be due to the fact that the first lagged autocorrelation of this model is stronger than the autocorrelation of the model (3.1) with $\rho=0.4$ but is weaker than the case with $\rho=0.85$. 
Table 1.A

SML-Model: Serial correlation only; no atate dependence True parameters: $\beta=1, \lambda=0.2$, and $\rho=0.4$

\begin{tabular}{|ccc|cccc|}
\hline$T$ & $n$ & $m$ & $\beta$ & $\lambda$ & $\rho$ & time \\
\hline & & & \multicolumn{4}{|c|}{ Bias unadjusted SML } \\
\hline & & & & & \\
8 & 200 & 15 & $0.9939(.0542)$ & $.1999(.0451)$ & $.3757(.0504)$ & 21.83 \\
15 & 200 & 15 & $0.9895(.0406)$ & $.2007(.0337)$ & $.3724(.0322)$ & 43.99 \\
30 & 200 & 15 & $0.9854(.0285)$ & $.1984(.0237)$ & $.3633(.0206)$ & 97.27 \\
50 & 200 & 15 & $0.9803(.0227)$ & $.1953(.0164)$ & $.3540(.0170)$ & 170.54 \\
100 & 200 & 15 & $0.9719(.0144)$ & $.1948(.0123)$ & $.3369(.0109)$ & 367.64 \\
& & & & & & \\
8 & 200 & 50 & $0.9999(.0549)$ & $.2010(.0453)$ & $.3905(.0503)$ & 74.09 \\
15 & 200 & 50 & $0.9955(.0412)$ & $.2019(.0339)$ & $.3876(.0328)$ & 151.01 \\
30 & 200 & 50 & $0.9943(.0288)$ & $.2002(.0240)$ & $.3842(.0216)$ & 337.24 \\
50 & 200 & 50 & $0.9904(.0232)$ & $.1975(.0164)$ & $.3782(.0176)$ & 570.69 \\
100 & 200 & 50 & $0.9824(.0146)$ & $.1971(.0123)$ & $.3635(.0108)$ & 1242.82 \\
& & & & & & \\
\hline & & & & Bies adjusted SML & \\
\hline & & & & & & \\
8 & 200 & 15 & $1.0006(.0551)$ & $.2014(.0453)$ & $.3929(.0517)$ & 22.04 \\
15 & 200 & 15 & $0.9969(.0414)$ & $.2023(.0341)$ & $.3905(.0332)$ & 44.40 \\
30 & 200 & 15 & $0.9930(.0291)$ & $.1998(.0239)$ & $.3812(.0212)$ & 98.16 \\
50 & 200 & 15 & $0.9871(.0231)$ & $.1966(.0166)$ & $.3702(.0175)$ & 172.05 \\
100 & 200 & 15 & $0.9749(.0145)$ & $.1954(.0124)$ & $.3452(.0110)$ & 370.56 \\
& & & & & \\
8 & 200 & 50 & $1.0024(.0552)$ & $.2015(.0454)$ & $.3968(.0509)$ & 74.80 \\
15 & 200 & 50 & $0.9986(.0416)$ & $.2025(.0341)$ & $.3948(.0335)$ & 152.39 \\
30 & 200 & 50 & $0.9985(.0291)$ & $.2010(.0241)$ & $.3935(.0223)$ & 340.09 \\
50 & 200 & 50 & $0.9951(.0235)$ & $.1984(.0165)$ & $.3888(.0183)$ & 575.67 \\
100 & 200 & 50 & $0.9850(.0147)$ & $.1976(.0123)$ & $.3705(.0109)$ & 1252.41 \\
& & & & & & \\
\hline
\end{tabular}

Table 1.B

SML-Model: Serial correlation only; no state dependence Root mean squared errors

\begin{tabular}{|ccc|ccc|ccc|}
\hline$T$ & $n$ & $m$ & $\beta$ & $\lambda$ & $\rho$ & $\beta$ & $\lambda$ & $\rho$ \\
\hline & & & \multicolumn{2}{|c|}{ Bias unadjusted SML } & \multicolumn{3}{|c|}{ Bias adjusted SML } \\
\hline \multirow{8}{*}{} & 200 & 15 & .0545 & .0450 & .0559 & .0550 & .0453 & .0521 \\
15 & 200 & 15 & .0419 & .0336 & .0423 & .0415 & .0341 & .0345 \\
30 & 200 & 15 & .0320 & .0237 & .0421 & .0299 & .0239 & .0283 \\
50 & 200 & 15 & .0300 & .0170 & .0490 & .0264 & .0169 & .0346 \\
100 & 200 & 15 & .0316 & .0134 & .0641 & .0290 & .0132 & .0559 \\
& & & & & & & & \\
8 & 200 & 50 & .0548 & .0453 & .0511 & .0552 & .0454 & .0509 \\
15 & 200 & 50 & .0414 & .0339 & .0351 & .0416 & .0341 & .0338 \\
30 & 200 & 50 & .0293 & .0239 & .0268 & .0291 & .0241 & .0232 \\
50 & 200 & 50 & .0251 & .0166 & .0280 & .0240 & .0166 & .0214 \\
100 & 200 & 50 & .0229 & .0126 & .0380 & .0210 & .0125 & .0314 \\
& & & & & & & & \\
\hline
\end{tabular}


Table 2.A

SML-Model: Serial correlation only; no state dependence

True parameters: $\beta=1, \lambda=0.2$, and $\rho=0.85$

\begin{tabular}{|ccc|cccc|}
\hline$T$ & $n$ & $m$ & $\beta$ & $\lambda$ & $\rho$ & time \\
\hline & & & \multicolumn{4}{|c|}{ Bias unadjusted SML } \\
\hline 8 & 200 & 15 & $0.9671(.0650)$ & $.1922(.0734)$ & $.8217(.0339)$ & 24.69 \\
15 & 200 & 15 & $0.9439(.0478)$ & $.1908(.0712)$ & $.8176(.0203)$ & 49.23 \\
30 & 200 & 15 & $0.9040(.0338)$ & $.1857(.0614)$ & $.7984(.0148)$ & 103.21 \\
50 & 200 & 15 & $0.8643(.0288)$ & $.1718(.0451)$ & $.7779(.0116)$ & 170.20 \\
100 & 200 & 15 & $0.8228(.0179)$ & $.1596(.0355)$ & $.7496(.0089)$ & 353.02 \\
& & & & & & \\
8 & 200 & 50 & $0.9867(.0656)$ & $.1966(.0739)$ & $.8379(.0322)$ & 84.54 \\
15 & 200 & 50 & $0.9763(.0498)$ & $.1965(.0724)$ & $.8384(.0186)$ & 165.56 \\
30 & 200 & 50 & $0.9486(.0360)$ & $.1944(.0639)$ & $.8241(.0140)$ & 353.36 \\
50 & 200 & 50 & $0.9081(.0291)$ & $.1811(.0464)$ & $.8056(.0112)$ & 561.33 \\
100 & 200 & 50 & $0.8572(.0186)$ & $.1685(.0377)$ & $.7743(.0084)$ & 1164.10 \\
& & & & & & \\
\hline & & & & Bias adjusted SML & \\
\hline & & & & & & \\
8 & 200 & 15 & $0.9862(.0669)$ & $.1956(.0748)$ & $.8364(.0334)$ & 24.90 \\
15 & 200 & 15 & $0.9611(.0492)$ & $.1937(.0731)$ & $.8273(.0200)$ & 49.64 \\
30 & 200 & 15 & $0.9159(.0344)$ & $.1878(.0629)$ & $.8039(.0146)$ & 104.11 \\
50 & 200 & 15 & $0.8718(.0290)$ & $.1729(.0460)$ & $.7813(.0114)$ & 171.71 \\
100 & 200 & 15 & $0.8233(.0180)$ & $.1595(.0359)$ & $.7499(.0090)$ & 355.94 \\
& & & & & & \\
8 & 200 & 50 & $0.9955(.0666)$ & $.1983(.0745)$ & $.8444(.0322)$ & 85.25 \\
15 & 200 & 50 & $0.9882(.0508)$ & $.1988(.0737)$ & $.8448(.0185)$ & 166.94 \\
30 & 200 & 50 & $0.9607(.0367)$ & $.1968(.0654)$ & $.8293(.0138)$ & 356.19 \\
50 & 200 & 50 & $0.9166(.0293)$ & $.1826(.0475)$ & $.8092(.0112)$ & 566.29 \\
100 & 200 & 50 & $0.8593(.0189)$ & $.1685(.0382)$ & $.7752(.0085)$ & 1173.70 \\
& & & & & & \\
\hline
\end{tabular}

Table 2.B
SML-Model: Serial correlation only; no state dependence

Root mean squared errors

\begin{tabular}{|ccc|ccc|ccc|}
\hline$T$ & $n$ & $m$ & $\beta$ & $\lambda$ & $\rho$ & $\beta$ & $\lambda$ & $\rho$ \\
\hline & & & \multicolumn{2}{|c|}{ Bias unadjusted SML } & \multicolumn{3}{|c|}{ Bias adjusted SML } \\
\hline \multirow{2}{*}{} & 200 & 15 & .0728 & .0737 & .0441 & .0682 & .0748 & .0360 \\
15 & 200 & 15 & .0737 & .0717 & .0382 & .0626 & .0733 & .0302 \\
30 & 200 & 15 & .1018 & .0629 & .0537 & .0909 & .0639 & .0483 \\
50 & 200 & 15 & .1387 & .0531 & .0731 & .1315 & .0533 & .0697 \\
100 & 200 & 15 & .1781 & .0537 & .1008 & .1776 & .0541 & .1005 \\
& & & & & & & & \\
8 & 200 & 50 & .0669 & .0739 & .0343 & .0666 & .0744 & .0326 \\
15 & 200 & 50 & .0551 & .0724 & .0219 & .0521 & .0736 & .0192 \\
30 & 200 & 50 & .0627 & .0640 & .0294 & .0537 & .0653 & .0248 \\
50 & 200 & 50 & .0964 & .0500 & .0458 & .0883 & .0505 & .0423 \\
100 & 200 & 50 & .1440 & .0491 & .0762 & .1420 & .0495 & .0753 \\
& & & & & & & & \\
\hline
\end{tabular}


Table 3.A

SML-Serially correlated True parameters: $\beta=1, \lambda=0.2, \sigma=\sqrt{0.5}=0.7071 . .$, and $\rho=0.4$

\begin{tabular}{|c|c|c|c|c|c|c|c|}
\hline$T$ & $n$ & $m$ & $\beta$ & $\lambda$ & $\sigma$ & $\boldsymbol{\rho}$ & time \\
\hline & & & \multicolumn{5}{|c|}{ Bias unadjusted SML } \\
\hline 8 & 200 & 15 & $0.9694(.0650)$ & $.1992(.0724)$ & $.6408(.1362)$ & $.4018(.0867)$ & 23.28 \\
\hline 15 & 200 & 15 & $0.9724(.0456)$ & $.1933(.0661)$ & $.6942(.0741)$ & $.3755(.0452)$ & 39.34 \\
\hline 30 & 200 & 15 & $0.9579(.0344)$ & $.1902(.0696)$ & $.7181(.0628)$ & $.3472(.0293)$ & 89.87 \\
\hline 50 & 200 & 15 & $0.9559(.0277)$ & $.1956(.0745)$ & $.7404(.0681)$ & $.3279(.0183)$ & 177.12 \\
\hline 100 & 200 & 15 & $0.9486(.0202)$ & $.1879(.1001)$ & $.7903(.0839)$ & $.2983(.0133)$ & 410.54 \\
\hline 8 & 200 & 50 & $0.9912(.0658)$ & $.2063(.0757)$ & $.6948(.0974)$ & $.3864(.0800)$ & 73.80 \\
\hline 15 & 200 & 50 & $0.9918(.0453)$ & $.1959(.0633)$ & $.7102(.0662)$ & $.3865(.0434)$ & 123.30 \\
\hline 30 & 200 & 50 & $0.9802(.0350)$ & $.2033(.0654)$ & $.7124(.0552)$ & $.3739(.0272)$ & 300.90 \\
\hline 50 & 200 & 50 & $0.9798(.0273)$ & $.2029(.0652)$ & $.7253(.0525)$ & $.3613(.0179)$ & 572.90 \\
\hline \multirow[t]{2}{*}{100} & 200 & 50 & $0.9715(.0178)$ & $.1869(.0827)$ & $.7521(.0692)$ & $.3342(.0128)$ & 1394.93 \\
\hline & & & \multicolumn{5}{|c|}{ Bias adjusted SML } \\
\hline 8 & 200 & 15 & $0.9884(.0672)$ & $.2030(.0743)$ & $.6906(.1302)$ & $.3938(.0906)$ & 23.40 \\
\hline 15 & 200 & 15 & $0.9864(.0468)$ & $.1958(.0682)$ & $.7143(.0739)$ & $.3843(.0466)$ & 39.76 \\
\hline 30 & 200 & 15 & $0.9682(.0348)$ & $.1920(.0718)$ & $.7297(.0643)$ & $.3598(.0297)$ & 90.73 \\
\hline 50 & 200 & 15 & $0.9637(.0282)$ & $.1968(.0765)$ & $.7482(.0701)$ & $.3387(.0184)$ & 178.60 \\
\hline 100 & 200 & 15 & $0.9512(.0208)$ & $.1880(.1009)$ & $.7926(.0849)$ & $.3031(.0133)$ & 413.55 \\
\hline 8 & 200 & 50 & $0.9989(.0666)$ & $.2082(.0764)$ & $.7092(.0942)$ & $.3844(.0811)$ & 74.54 \\
\hline 15 & 200 & 50 & $0.9989(.0460)$ & $.1975(.0641)$ & $.7151(.0659)$ & $.3938(.0443)$ & 124.73 \\
\hline 30 & 200 & 50 & $0.9878(.0356)$ & $.2054(.0666)$ & $.7158(.0560)$ & $.3860(.0280)$ & 303.82 \\
\hline 50 & 200 & 50 & $0.9871(.0277)$ & $.2042(.0675)$ & $.7297(.0539)$ & $.3741(.0181)$ & 577.72 \\
\hline 100 & 200 & 50 & $0.9743(.0179)$ & $.1867(.0840)$ & $.7545(.0703)$ & $.3414(.0130)$ & 1404.82 \\
\hline
\end{tabular}

Table 3.B

SML-Serially correlated disturbances; random component; no state dependence Root mean squared exror

\begin{tabular}{|ccc|cccc|cccc|}
\hline$T$ & $n$ & $m$ & $\beta$ & $\lambda$ & $\sigma$ & $\rho$ & $\beta$ & $\lambda$ & $\sigma$ & $\rho$ \\
\hline & & & \multicolumn{3}{|c|}{ Bias unadjusted SML } & \multicolumn{3}{|c|}{ Bias adjusted SML } \\
\hline \multirow{2}{*}{} & 200 & 15 & .0717 & .0722 & .1513 & .0866 & .0681 & .0742 & .1311 & .0906 \\
15 & 200 & 15 & .0532 & .0663 & .0751 & .0514 & .0487 & .0682 & .0741 & .0490 \\
30 & 200 & 15 & .0544 & .0702 & .0637 & .0604 & .0471 & .0722 & .0680 & .0500 \\
50 & 200 & 15 & .0520 & .0745 & .0757 & .0744 & .0459 & .0765 & .0812 & .0640 \\
100 & 200 & 15 & .0552 & .1006 & .1180 & .1025 & .0531 & .1014 & .1204 & .0978 \\
& & & & & & & & & \\
8 & 200 & 50 & .0663 & .0758 & .0980 & .0810 & .0665 & .0767 & .0941 & .0825 \\
15 & 200 & 50 & .0460 & .0633 & .0662 & .0454 & .0459 & .0641 & .0663 & .0446 \\
30 & 200 & 50 & .0402 & .0654 & .0553 & .0377 & .0376 & .0668 & .0566 & .0312 \\
50 & 200 & 50 & .0339 & .0652 & .0554 & .0426 & .0305 & .0676 & .0583 & .0316 \\
100 & 200 & 50 & .0336 & .0836 & .0825 & .0670 & .0313 & .0849 & .0846 & .0601 \\
& & & & & & & & & & \\
\hline
\end{tabular}




\section{Markov Model}

4.1 Markov Model with Serially Correlated Disturbances

The Markov dynamic choice model considered in the experiment is

$$
y_{i t}^{*}=\beta x_{i t}+\lambda d_{i, t-1}+\epsilon_{i t}
$$

where

$$
c_{i t}=\rho c_{i, t-1}+w_{i t}
$$

and $w_{i t}$ are i.i.d. $N(0,1)$. The stochastic process starts at the initial period with $\epsilon_{i 0}=0$ and $d_{i 0}=0$ for all i. This model is a first order Markov model as choices made last period are the only prior choices relevant to current choice. The simulated log likelihood function for this model is

$$
\mathcal{L}=\sum_{i=1}^{n} \ln \left\{\frac{1}{m} \sum_{j=1}^{m} \prod_{t=1}^{T} \Phi\left[D_{i t}\left(\beta x_{i t}+\lambda d_{i, t-1}+\rho \epsilon_{i, t-1}^{(j)}\right)\right]\right\} .
$$

This model differs from the model in (3.1) in that the $\lambda$ represents the effect of true state dependence. The $x$ variable is generated by the same stochastic process before.

Tables 4.A and 4.B report simulated likelihood estimates and their root mean square errors for the case where true parameters are set to $\beta=1, \lambda=0.2$, and $\rho=0.4$. With a sample of size $n=200$ and $m=15$, biases of the SMLE of $\beta$ are small. But there are upward biases in the SMLE of $\lambda$. The SMLE of $\rho$ are biased downward. So the dynamic effect can be over stated but the serial correlation of disturbances can be underestimated. Standard errors of all the SMLE decrease as panels become longer. For the longest panel with $T=100$, biases dominate standard errors and their root mean square errors become larger than the root mean square errors for panels with shorter periods. With $m=50$, biases of the SMLE of $\lambda$ and $\rho$ have been substantially reduced. The bias correction procedure can also substantially reduce biases of the SMLE. By solely increasing the sample size $n$ (from 200 to 500 ) but without increasing the number of simulated random variables $m$, biases can not be reduced as predicted by theory. Overall, estimates of this model are reasonably good for panels with $T$ up to 50 . With $m=50$, bias-corrected estimates have small root mean square errors among all the estimates. Comparing these estimates with estimates of the regression model without state dependence in Tables 1.A and 1.B, standard errors and root mean square errors of estimates of the Markov dynamic model are larger. When small $m(m=15)$, is utilized, the estimate of the dynamic effect of the Markov model is more biased.
Tables 5.A and 5.B provide estimates for the case with a high autocorrelation $\rho=0.85$. Biases and standard errors of the SMLE for both $\beta$ and $\lambda$ become larger, even though standard errors for $\rho$ are smaller. With the bias-correction procedure and $m=50$, estimates can be quite accurate for shorter panels with $T$ up to 15. Biases and root mean square errors tend to be larger for panels with $T$ equal to 30 or more.

4.2 Markov Model with Unobserved Heterogeneity and Serially Correlated Disturbances

The Markov dynamic choice model considered for Monte Carlo study in this rection is

$$
y_{i t}^{*}=\beta x_{i t}+\lambda d_{i, t-1}+\sigma \xi_{i}+\epsilon_{i t},
$$

where

$$
\epsilon_{i t}=\rho \epsilon_{i, t-1}+w_{i t},
$$

and $\xi_{i}$ and $w_{i t}$ are i.i.d. $N(0,1)$. The stochastic process starts at the initial period with $\epsilon_{i 0}=0$ and $d_{i 0}=0$ for all $i$. The simulated log likelihood function for this model is

$$
\mathcal{L}=\sum_{i=1}^{n} \ln \left\{\frac{1}{m} \sum_{j=1}^{m} \prod_{t=1}^{T} \Phi\left[D_{i t}\left(\beta x_{i t}+\lambda d_{i, t-1}+\sigma \xi_{i}^{(j)}+\rho \epsilon_{i, t-1}^{(j)}\right)\right]\right\} .
$$

Sample dats are generated with $\beta=1, \lambda=0.2, \sigma^{2}=0.5$, and $\rho=0.4$.

The SMLE and their root mean equare errors are reported on Tables 6.A and 6.B. The SMLE of $\beta$ are biased downward, biases of the dynamic effect $\lambda$ are upward, and estimates of $\rho$ are biased downward. The magnitude of bias increases as the length of the panel becomes longer. The SMLE of $\sigma$ with omall $m=15$ are biased downward for short panels but biases become upward for long panels. Directions of bias of the SMLE for $\beta, \lambda$ and $\rho$ are similar to the Markov model (4.1) without unobserved heterogeneity. Biases for $\beta$ for the Markov model with additional unobserved heterogeneity are slightly larger. Biasea for $\rho$ are much more severe when $\boldsymbol{T}$ are 30 or longer. Bisses of the SMLE of the dynamic effect $\lambda$ are aleo quite severe for $T$ as long as 30 or longer. By increasing $m$ to 50 , biases of estimates are reduced. Bias-corrected eatimates have smaller biases. With $m=50$, biases of bias-corrected estimates for all the parameters seem quite small for $T$ up to 15. In terms of root mean squares errors, estimates are improved with $T$ up to 30 . When $T$ becomes long as 50 or 100, root mean squares errors of estimates can be larger than the ones with shorter $T$. By increasing $m$ to a large 100 , biases and root mean squares errore can further be reduced but the time cost is double. Bias-corrected estimates have slightly larger standard errors but their root mean equare errors are, in general, smaller. In conclusion, for this model, bis-corrected estimates with $m=50$ can be reasonably good for $T$ up to 30 . 
Table 4.A

SML-aerial correlated disturbances; Markov state dependence

True parametern: $\beta=1, \lambda=0.2$, and $\rho=0.4$

\begin{tabular}{|ccc|cccc|}
\hline$T$ & $n$ & $m$ & $\beta$ & $\lambda$ & $\rho$ & time \\
\hline & & & \multicolumn{4}{|c|}{ Bias unadjusted SML } \\
\hline 8 & 200 & 15 & $0.9928(.0562)$ & $.2217(.0754)$ & $.3682(.0622)$ & 20.04 \\
15 & 200 & 15 & $0.9853(.0425)$ & $.2293(.0520)$ & $.3609(.0373)$ & 40.21 \\
30 & 200 & 15 & $0.9799(.0287)$ & $.2339(.0341)$ & $.3505(.0260)$ & 81.65 \\
50 & 200 & 15 & $0.9743(.0235)$ & $.2391(.0270)$ & $.3382(.0191)$ & 146.25 \\
100 & 200 & 15 & $0.0648(.0145)$ & $.2598(.0185)$ & $.3153(.0141)$ & 318.58 \\
& & & & & \\
8 & 200 & 50 & $1.0007(.0562)$ & $.2076(.0768)$ & $.3890(.0626)$ & 67.77 \\
15 & 200 & 50 & $0.9939(.0434)$ & $.2138(.0536)$ & $.3828(.0388)$ & 137.84 \\
30 & 200 & 50 & $0.9920(.0293)$ & $.2128(.0350)$ & $.3804(.0277)$ & 277.19 \\
50 & 200 & 50 & $0.9877(.0240)$ & $.2161(.0281)$ & $.3713(.0201)$ & 483.74 \\
100 & 200 & 50 & $0.9780(.0144)$ & $.2368(.0193)$ & $.3497(.0142)$ & 1062.70 \\
& & & & & & \\
15 & 500 & 15 & $0.9828(.0233)$ & $.2289(.0300)$ & $.3622(.0235)$ & 101.65 \\
30 & 500 & 15 & $0.9795(.0161)$ & $.2340(.0206)$ & $.3515(.0168)$ & 239.84 \\
& & & & & & \\
\hline & & & & Bias adjusted SML & \\
\hline & & & & & & \\
8 & 200 & 15 & $1.0020(.0576)$ & $.2057(.0766)$ & $.3922(.0640)$ & 20.27 \\
15 & 200 & 15 & $0.9953(.0436)$ & $.2123(.0533)$ & $.3862(.0387)$ & 40.62 \\
30 & 200 & 15 & $0.9899(.0295)$ & $.2170(.0352)$ & $.3753(.0270)$ & 82.49 \\
50 & 200 & 15 & $0.0833(.0238)$ & $.2240(.0277)$ & $.3605(.0197)$ & 147.71 \\
100 & 200 & 15 & $0.9691(.0148)$ & $.2521(.0187)$ & $.3275(.0143)$ & 321.51 \\
& & & & \\
8 & 200 & 50 & $1.0042(.0568)$ & $.2014(.0774)$ & $.3979(.0633)$ & 68.48 \\
15 & 200 & 50 & $0.9982(.0440)$ & $.2063(.0544)$ & $.3933(.0397)$ & 139.23 \\
30 & 200 & 50 & $0.9977(.0298)$ & $.2030(.0358)$ & $.3939(.0286)$ & 280.02 \\
50 & 200 & 50 & $0.9940(.0244)$ & $.2052(.0288)$ & $.3865(.0211)$ & 488.51 \\
100 & 200 & 50 & $0.9819(.0147)$ & $.2297(.0195)$ & $.3603(.0146)$ & 1072.31 \\
& & & & & & \\
15 & 500 & 15 & $0.9927(.0240)$ & $.2121(.0307)$ & $.3870(.0246)$ & 102.74 \\
30 & 500 & 15 & $0.9894(.0164)$ & $.2174(.0212)$ & $.3760(.0174)$ & 242.01 \\
& & & & & & \\
\hline
\end{tabular}

Table 4.B

Tablo 4.B Red mean eques; Markov

\begin{tabular}{|ccc|ccc|ccc|}
\hline$T$ & $n$ & $m$ & $\beta$ & $\lambda$ & $\rho$ & $\beta$ & $\lambda$ & $\rho$ \\
\hline & & & \multicolumn{2}{|c|}{ Bias unadjusted SML } & \multicolumn{3}{|c|}{ Bias adjusted SML } \\
\hline \multirow{2}{*}{} & 200 & 15 & .0565 & .0783 & .0697 & .0575 & .0767 & .0644 \\
15 & 200 & 15 & .0449 & .0596 & .0540 & .0438 & .0546 & .0410 \\
30 & 200 & 15 & .0350 & .0481 & .0559 & .0311 & .0390 & .0366 \\
50 & 200 & 15 & .0348 & .0475 & .0647 & .0290 & .0367 & .0441 \\
100 & 200 & 15 & .0381 & .0626 & .0859 & .0343 & .0553 & .0739 \\
& & & & & & & & \\
8 & 200 & 50 & .0561 & .0770 & .0634 & .0569 & .0772 & .0632 \\
15 & 200 & 50 & .0438 & .0553 & .0424 & .0440 & .0546 & .0402 \\
30 & 200 & 50 & .0303 & .0372 & .0339 & .0298 & .0358 & .0292 \\
50 & 200 & 50 & .0270 & .0323 & .0350 & .0251 & .0292 & .0250 \\
100 & 200 & 50 & .0263 & .0415 & .0523 & .0232 & .0355 & .0423 \\
\end{tabular}


Table 3.A

SML-Serially correlated disturbances; Markov atate dependence True parameters: $\beta=1, \lambda=0.2$, and $\rho=0.85$

\begin{tabular}{|ccc|cccc|}
\hline$T$ & $n$ & $m$ & $\beta$ & $\lambda$ & $\rho$ & time \\
\hline & & & \multicolumn{4}{|c|}{ Bias unadjusted SML } \\
\hline \\
8 & 200 & 15 & $0.9744(.0683)$ & $.2231(.0965)$ & $.8179(.0387)$ & 24.42 \\
15 & 200 & 15 & $0.9478(.0501)$ & $.2358(.0695)$ & $.8118(.0232)$ & 47.40 \\
30 & 200 & 15 & $0.9077(.0357)$ & $.2626(.0520)$ & $.7892(.0182)$ & 104.58 \\
50 & 200 & 15 & $0.8689(.0282)$ & $.2895(.0413)$ & $.7621(.0157)$ & 188.52 \\
100 & 200 & 15 & $0.8277(.0192)$ & $.3455(.0349)$ & $.7222(.0114)$ & 386.83 \\
& & & & & & \\
8 & 200 & 50 & $0.9943(.0684)$ & $.2093(.0948)$ & $.8371(.0350)$ & 83.45 \\
15 & 200 & 50 & $0.9794(.0506)$ & $.2135(.0689)$ & $.8366(.0203)$ & 159.61 \\
30 & 200 & 50 & $0.9507(.0372)$ & $.2322(.0476)$ & $.8202(.0159)$ & 346.88 \\
50 & 200 & 50 & $0.9116(.0295)$ & $.2520(.0380)$ & $.7975(.0136)$ & 627.23 \\
100 & 200 & 50 & $0.8647(.0201)$ & $.3094(.0340)$ & $.7566(.0104)$ & 1352.99 \\
& & & & & & \\
\hline & & & & Bias adjusted SML & \\
\hline & & & & & & \\
8 & 200 & 15 & $0.9932(.0706)$ & $.2093(.0973)$ & $.8355(.0377)$ & 24.64 \\
15 & 200 & 15 & $0.9642(.0515)$ & $.2248(.0697)$ & $.8231(.0225)$ & 47.84 \\
30 & 200 & 15 & $0.9194(.0365)$ & $.2559(.0525)$ & $.7960(.0177)$ & 105.46 \\
50 & 200 & 15 & $0.8765(.0283)$ & $.2851(.0416)$ & $.7605(.0153)$ & 189.94 \\
100 & 200 & 15 & $0.8290(.0195)$ & $.3422(.0350)$ & $.7235(.0114)$ & 389.84 \\
& & & & & \\
8 & 200 & 50 & $1.0028(.0695)$ & $.2034(.0953)$ & $.8446(.0347)$ & 84.16 \\
15 & 200 & 50 & $0.9907(.0516)$ & $.2066(.0692)$ & $.8439(.0200)$ & 159.61 \\
30 & 200 & 50 & $0.9622(.0379)$ & $.2259(.0477)$ & $.8263(.0154)$ & 346.88 \\
50 & 200 & 50 & $0.9199(.0297)$ & $.2474(.0384)$ & $.8018(.0134)$ & 631.98 \\
100 & 200 & 50 & $0.8674(.0202)$ & $.3059(.0342)$ & $.7584(.0104)$ & 1362.58 \\
& & & & & & \\
\hline
\end{tabular}

Table 5.B

SML-Serially correlated disturbances; Markov atate dependence Root mean squared errors

\begin{tabular}{|ccc|ccc|ccc|}
\hline$r$ & $n$ & $m$ & $\beta$ & $\lambda$ & $\rho$ & $\beta$ & $\lambda$ & $\rho$ \\
\hline & & & \multicolumn{2}{|c|}{ Biea unedjusted SML } & \multicolumn{3}{|c|}{ Bia sdjusted SML } \\
\hline \multirow{2}{*}{} & 200 & 15 & .0729 & .0991 & .0502 & .0708 & .0976 & .0403 \\
15 & 200 & 15 & .0723 & .0781 & .0447 & .0626 & .0738 & .0351 \\
30 & 200 & 15 & .0989 & .0813 & .0634 & .0885 & .0766 & .0568 \\
50 & 200 & 15 & .1341 & .0985 & .0893 & .1267 & .0947 & .0849 \\
100 & 200 & 15 & .1734 & .1496 & .1283 & .1721 & .1464 & .1270 \\
& & & & & & & & \\
8 & 200 & 50 & .0686 & .0951 & .0372 & .0694 & .0952 & .0351 \\
15 & 200 & 50 & .0545 & .0701 & .0243 & .0523 & .0694 & .0209 \\
30 & 200 & 50 & .0617 & .0574 & .0338 & .0535 & .0542 & .0283 \\
50 & 200 & 50 & .0932 & .0644 & .0542 & .0855 & .0609 & .0500 \\
100 & 200 & 50 & .1368 & .1146 & .0940 & .1342 & .1113 & .0922 \\
& & & & & & & & \\
\hline
\end{tabular}


Table 6.A

SML-Serially correlated disturbances; random component; Markov state dependence True parameters: $\beta=1, \lambda=0.2, \sigma=\sqrt{0.5}=0.7071 . .$, and $\rho=0.4$

\begin{tabular}{|c|c|c|c|c|c|c|c|}
\hline$T$ & $n$ & $m$ & $\beta$ & $\lambda$ & $\sigma$ & $p$ & time \\
\hline & & & \multicolumn{5}{|c|}{ Bias unadjusted SML } \\
\hline 8 & 200 & 15 & $0.9662(.0637)$ & $.2317(.1081)$ & $.6299(.1358)$ & $.3876(.1124)$ & 25.24 \\
\hline 15 & 200 & 15 & $0.9654(.0448)$ & $.2667(.0827)$ & $.6766(.0759)$ & $.3427(.0692)$ & 44.65 \\
\hline 30 & 200 & 15 & $0.9516(.0347)$ & $.3216(.0652)$ & $.6904(.0622)$ & $.2905(.0464)$ & 91.16 \\
\hline 50 & 200 & 15 & $0.9510(.0269)$ & $.3775(.0562)$ & $.7107(.0640)$ & $.2525(.0337)$ & 157.31 \\
\hline 100 & 200 & 15 & $0.9459(.0212)$ & $.4817(.0526)$ & $.7662(.0799)$ & $.1860(.0266)$ & 353.14 \\
\hline 8 & 200 & 50 & $0.9884(.0660)$ & $.2224(.1073)$ & $.6851(.0963)$ & $.3787(.1055)$ & 81.35 \\
\hline 15 & 200 & 50 & $0.9876(.0458)$ & $.2227(.0768)$ & $.6978(.0712)$ & $.3795(.0620)$ & 152.19 \\
\hline 30 & 200 & 50 & $0.9763(.0344)$ & $.2598(.0637)$ & $.6946(.0575)$ & $.3442(.0464)$ & 334.48 \\
\hline 50 & 200 & 50 & $0.9775(.0268)$ & $.3030(.0541)$ & $.6956(.0532)$ & $.3153(.0341)$ & 558.50 \\
\hline 100 & 200 & 50 & $0.9713(.0179)$ & $.3848(.0400)$ & $.7168(.0663)$ & $.2610(.0214)$ & 1178.51 \\
\hline 8 & 200 & 100 & $0.9927(.0658)$ & $.2167(.1056)$ & $.6942(.0927)$ & $.3819(.1027)$ & 161.69 \\
\hline 15 & 200 & 100 & $0.9933(.0467)$ & $.2120(.0783)$ & $.7021(.0714)$ & $.3890(.0620)$ & 300.13 \\
\hline 30 & 200 & 100 & $0.9839(.0334)$ & $.2337(.0619)$ & $.6975(.0562)$ & $.3671(.0432)$ & 667.17 \\
\hline 50 & 200 & 100 & $0.9868(.0269)$ & $.2640(.0513)$ & $.6972(.0510)$ & $.3462(.0334)$ & 1151.58 \\
\hline \multirow[t]{2}{*}{100} & 200 & 100 & $0.9790(.0180)$ & $.3382(.0387)$ & $.7027(.0542)$ & $.2970(.0201)$ & 2378.40 \\
\hline & & & \multicolumn{5}{|c|}{ Bias adjusted SML } \\
\hline 8 & 200 & 15 & $0.9871(.0661)$ & $.2146(.1111)$ & $.6819(.1314)$ & $.3880(.1171)$ & 25.46 \\
\hline 15 & 200 & 15 & $0.9812(.0459)$ & $.2435(.0849)$ & $.7000(.0759)$ & $.3610(.0715)$ & 45.07 \\
\hline 30 & 200 & 15 & $0.9633(.0353)$ & $.2990(.0673)$ & $.7039(.0636)$ & $.3120(.0479)$ & 92.02 \\
\hline 50 & 200 & 15 & $0.9602(.0276)$ & $.3604(.0575)$ & $.7197(.0655)$ & $.2708(.0348)$ & 158.78 \\
\hline 100 & 200 & 15 & $0.9493(.0214)$ & $.4747(.0533)$ & $.7688(.0810)$ & $.1947(.0274)$ & 356.08 \\
\hline 8 & 200 & 50 & $0.9969(.0670)$ & $.2147(.1087)$ & $.7007(.0938)$ & $.3808(.1075)$ & 82.09 \\
\hline 15 & 200 & 50 & $0.9958(.0465)$ & $.2062(.0781)$ & $.7057(.0714)$ & $.3944(.0633)$ & 153.63. \\
\hline 30 & 200 & 50 & $0.9847(.0349)$ & $.2346(.0652)$ & $.7020(.0587)$ & $.3668(.0477)$ & 337.42 \\
\hline 50 & 200 & 50 & $0.9855(.0273)$ & $.2749(.0555)$ & $.7034(.0550)$ & $.3401(.0347)$ & 563.44 \\
\hline 100 & 200 & 50 & $0.9747(.0180)$ & $.3667(.0400)$ & $.7203(.0672)$ & $.2768(.0217)$ & 1188.43 \\
\hline 8 & 200 & 100 & $0.9973(.0664)$ & $.2123(.1063)$ & $.7017(.0915)$ & $.3836(.1038)$ & 163.14 \\
\hline 15 & 200 & 100 & $0.9982(.0471)$ & $.2016(.0791)$ & $.7058(.0714)$ & $.3986(.0629)$ & 302.97 \\
\hline 30 & 200 & 100 & $0.9901(.0338)$ & $.2144(.0634)$ & $.7023(.0573)$ & $.3843(.0443)$ & 683.03 \\
\hline 50 & 200 & 100 & $0.9934(.0276)$ & $.2385(.0525)$ & $.7042(.0525)$ & $.3678(.0342)$ & 1161.44 \\
\hline 100 & 200 & 100 & $0.9822(.0182)$ & $.3183(.0386)$ & $.7069(.0552)$ & $.3132(.0202)$ & 2398.21 \\
\hline
\end{tabular}

Table 6.B

SML-Serially correlated disturbances; random component; Markov otate dependence Root mean squared error

\begin{tabular}{|ccc|cccc|cccc|}
\hline$T$ & $n$ & $m$ & $\beta$ & $\lambda$ & $\sigma$ & $\rho$ & $\beta$ & $\lambda$ & $\sigma$ & $\rho$ \\
\hline & & & \multicolumn{3}{|c|}{ Bias unadjuated SML } & \multicolumn{3}{|c|}{ Bias adjuotod SML } \\
\hline \multirow{8}{*}{} & 200 & 15 & .0720 & .1124 & .1560 & .1129 & .0672 & .1118 & .1335 & .1175 \\
15 & 200 & 15 & .0565 & .1062 & .0817 & .0897 & .0496 & .0952 & .0761 & .0814 \\
30 & 200 & 15 & .0596 & .1379 & .0643 & .1189 & .0509 & .1197 & .0636 & .1002 \\
50 & 200 & 15 & .0559 & .1862 & .0639 & .1513 & .0484 & .1704 & .0686 & .1338 \\
100 & 200 & 15 & .0581 & .2865 & .0993 & .2156 & .0550 & .2799 & .1017 & .2072 \\
& & & & & & & & & & \\
8 & 200 & 50 & .0669 & .1094 & .0986 & .1075 & .0669 & .1095 & .0939 & .1090 \\
15 & 200 & 50 & .0473 & .0800 & .0716 & .0652 & .0466 & .0782 & .0713 & .0635 \\
30 & 200 & 50 & .0417 & .0873 & .0588 & .0725 & .0380 & .0737 & .0589 & .0580 \\
50 & 200 & 50 & .0349 & .1163 & .0544 & .0913 & .0309 & .0931 & .0550 & .0692 \\
100 & 200 & 50 & .0338 & .1891 & .0669 & .1406 & .0310 & .1714 & .0683 & .1251 \\
& & & & &. & & & & & \\
8 & 200 & 100 & .0661 & .1067 & .0934 & .1041 & .0683 & .1068 & .0915 & .1049 \\
15 & 200 & 100 & .0471 & .0791 & .0714 & .0629 & .0471 & .0790 & .0713 & .0628 \\
30 & 200 & 100 & .0370 & .0704 & .0570 & .0543 & .0351 & .0649 & .0574 & .0469 \\
50 & 200 & 100 & .0299 & .0819 & .0519 & .0633 & .0283 & .0650 & .0525 & .0469 \\
100 & 200 & 100 & .0277 & .1435 & .0543 & .1050 & .0254 & .1244 & .0551 & .0891 \\
& & & & & & & & & & \\
\hline
\end{tabular}




\section{Generalized Polya Modele}

5.1 Polya Models with Serially Correlated Disturbances

The generalized Polya model with a depreciation factor $\delta$ is considered for our Monte Carlo experiment. The Polya model is apecified as

$$
y_{i t}^{*}=x_{i t} \beta+\lambda \sum_{j=1}^{j} \delta^{j-1} d_{i, t-j}+\epsilon_{i t}
$$

and

$$
\epsilon_{i t}=\rho \epsilon_{i, t-1}+w_{i t},
$$

where $w_{i t}$ are i.i.d. $N(0,1)$. The initial conditions are $c_{i 0}=0$ and $d_{i 0}=0$ for all $i$. In this model, the entire history of the process is relevant to current decision making. The simulated log likelihood function for this model is

$$
\mathcal{L}=\sum_{i=1}^{n} \ln \left\{\frac{1}{m} \sum_{j=1}^{m} \prod_{t=1}^{T} \Phi\left[D_{i t}\left(\beta x_{i t}+\lambda \sum_{j=1}^{l} \delta^{j} d_{i, t-1}+\rho_{i, t-1}^{(j)}\right)\right]\right\}
$$

For comparison purpose, the discount factor $\delta$ is assumed to be a known constant and is set to be 0.7. Sample data are generated with parameters $\beta=1, \lambda=0.2$, and $\rho=0.4$.

The SMLE and the bias-corrected estimates for this model are reported in Tables 7.A and 7.B. We have experimented with $T=8,15,30$, and 50. Biases of all the SMLE of $\beta$ and $\lambda$ are very small even for $m=15$. There are some downward biases for the SMLE of $\rho$ for large $T$ with $m=15$. These biases can be easily reduced by increasing $m$ to 50 or by the bias correction procedure. Even with $m=15$, the bias corrected estimates are quit good. Estimates of the dynamic effect $\lambda$ are accurate for all these $T$. Comparing estimates of this model with corresponding estimates of the Markov model in Table 4, the Polya model can be better estimated. Estimates of the dynamic effect $\lambda$ not only have omall biases but also have much omaller standard errors. The generalized Polya model has apparently much stronger state dependence property than the Markov property. That may explain the excellent statistical properties of these estimates. On the other hand, the computational time coot for this model is relatively more expensive. The additional time cost is due to the need of computing more tail probabilities in this model.

5.2 Polya Models with Unobserved Heterogeneity and Serially Correlated Disturbances

With a random component $\boldsymbol{\xi}$ to capture unobserved heterogeneity, the generalized Polya model for our experiment is specified as

$$
y_{i t}^{i}=x_{i t} \beta+\lambda \sum_{j=1}^{t} \delta^{j-1} d_{i, t-j}+\sigma \xi_{i}+\epsilon_{i t,}
$$

and

$$
\epsilon_{i t}=\rho \epsilon_{i, t-1}+w_{i t},
$$

where $\xi_{i}$ and $w_{i t}$ are i.i.d. $N(0,1)$. The simulated log likelihood function for this model is

$$
\mathcal{L}=\sum_{i=1}^{n} \ln \left\{\frac{1}{m} \sum_{j=1}^{m} \prod_{t=1}^{T}\left[D_{i t}\left(\beta x_{i t}+\lambda \sum_{j=1}^{\dot{S}} \delta^{j} d_{i, t-1}+\sigma \xi_{i}^{(j)}+\rho \epsilon_{i, t-1}^{(j)}\right)\right]\right\} .
$$

Tables 8.A and 8.B report the SMLE and bias-corrected estimates and their root mean equare errore. There are some downward biases in the SMLE of $\beta$ and $\rho$ and upward biases for estimating the dynamic effect $\lambda$. These biases are not large. Estimates of $\sigma$ are biased downward for small $T$. There seems to be a trado-off between estimates of $\sigma$ and $\rho$. As $T$ becomes longer, the value of estimates of $\sigma$ increases but the value of estimates of $\rho$ decreases. For $T=50$ and $m=15$, the bias of $\rho$ is 25 percent. The bias-correction procedure reduces biases. With $m=\mathbf{5 0}$ and the bisa-correction procedure, estimates of this model are reasonably good for $T$ up to 50 . Comparing these eatimates with the corresponding estimates of the Markov model with unobserved heterogeneity in Table 6 , the dynamic effect $\lambda$ and the serial correlation coefficient $\rho$ in the Polya model can be much better estimated. They have omall biases and smaller atandard errors. This may be due to the stronger dynamic effect in this model. With the additional unobserved heterogeneity, eatimates are leses precise when compared with estimates of the Polya model without unobserved heterogeneity in (5.1). The time cost for estimation, however, turns out to be less due to the fact that choice probabilities are now less extreme as an additional disturbance is introduced. 
Table 7.A

7. Ances; the Polya model True parameters: $\beta=1, \lambda=0.2$, and $\rho=0.4$

\begin{tabular}{|c|c|c|c|c|c|c|}
\hline$T$ & $n$ & $m$ & $\beta$ & $\lambda$ & $\boldsymbol{\rho}$ & time \\
\hline & & & \multicolumn{4}{|c|}{ Bias unadjusted SML } \\
\hline 8 & 200 & 15 & $0.9949(.0544)$ & $.2054(.0343)$ & $.3821(.0539)$ & 31.32 \\
\hline 15 & 200 & 15 & $0.9921(.0450)$ & $.2056(.0209)$ & $.3801(.0348)$ & 67.58 \\
\hline 30 & 200 & 15 & $0.9856(.0297)$ & $.2047(.0130)$ & $.3709(.0226)$ & 152.63 \\
\hline 50 & 200 & 15 & $0.9788(.0232)$ & $.2022(.0082)$ & $.3547(.0197)$ & 276.65 \\
\hline 8 & 200 & 50 & $1.0026(.0547)$ & $.2018(.0351)$ & $.4007(.0537)$ & 107.75 \\
\hline 15 & 200 & 50 & $1.0000(.0459)$ & $.2031(.0215)$ & $.3979(.0361)$ & 231.64 \\
\hline 30 & 200 & 50 & $0.9970(.0310)$ & $.2028(.0132)$ & $.3953(.0232)$ & 505.92 \\
\hline \multirow[t]{2}{*}{50} & 200 & 50 & $0.9917(.0235)$ & $.2002(.0084)$ & $.3832(.0208)$ & 928.09 \\
\hline & & & \multicolumn{4}{|c|}{ Bias adjusted SML } \\
\hline 8 & 200 & 15 & $1.0036(.0557)$ & $.2016(.0348)$ & $.4023(.0559)$ & 31.55 \\
\hline 15 & 200 & 15 & $1.0013(.0460)$ & $.2032(.0213)$ & $.4011(.0363)$ & 68.02 \\
\hline 30 & 200 & 15 & $0.9950(.0303)$ & $.2029(.0132)$ & $.3914(.0236)$ & 153.48 \\
\hline 50 & 200 & 15 & $0.9872(.0238)$ & $.2010(.0084)$ & $.3731(.0204)$ & 278.10 \\
\hline 8 & 200 & 50 & $1.0059(.0552)$ & $.2003(.0353)$ & $.4082(.0544)$ & 108.48 \\
\hline 15 & 200 & 50 & $1.0040(.0465)$ & $.2020(.0217)$ & $.4066(.0370)$ & 233.15 \\
\hline 30 & 200 & 50 & $1.0022(.0314)$ & $.2016(.0133)$ & $.4062(.0242)$ & 508.90 \\
\hline 50 & 200 & 50 & $0.9977(.0241)$ & $.1991(.0086)$ & $.3957(.0217)$ & $\mathbf{9 3 2 . 8 5}$ \\
\hline
\end{tabular}

Table 7.B

Rrelated disturbances; the Polya mode

\begin{tabular}{|ccc|ccc|ccc|}
\hline$T$ & $n$ & $m$ & $\beta$ & $\lambda$ & $\rho$ & $\beta$ & $\lambda$ & $\rho$ \\
\hline & & & \multicolumn{2}{|c|}{ Bias unadjusted SML } & \multicolumn{3}{|c|}{ Bias adjusted SML } \\
\hline $\mathbf{8}$ & 200 & 15 & .0546 & .0347 & .0567 & .0557 & .0348 & .0558 \\
15 & 200 & 15 & .0456 & .0216 & .0400 & .0459 & .0215 & .0362 \\
30 & 200 & 15 & .0330 & .0138 & .0368 & .0306 & .0135 & .0250 \\
50 & 200 & 15 & .0314 & .0084 & .0493 & .0270 & .0084 & .0338 \\
& & & & & & & & \\
8 & 200 & 50 & .0547 & .0351 & .0536 & .0554 & .0352 & .0550 \\
15 & 200 & 50 & .0459 & .0217 & .0361 & .0465 & .0218 & .0375 \\
30 & 200 & 50 & .0311 & .0134 & .0237 & .0315 & .0134 & .0249 \\
50 & 200 & 50 & .0249 & .0084 & .0267 & .0241 & .0086 & .0221 \\
& & & & & & & & \\
\hline
\end{tabular}

Table 8.A

SML-Serially correlated disturbecto True parameters: $\beta=1, \lambda=0.2, \sigma=\sqrt{0.5}$, and $\rho=0.4$

\begin{tabular}{|c|c|c|c|c|c|c|c|}
\hline$T$ & $n$ & $\boldsymbol{m}$ & $\boldsymbol{\beta}$ & $\lambda$ & $\sigma$ & $\boldsymbol{\rho}$ & time \\
\hline & & & \multicolumn{5}{|c|}{ Bias unadjusted SML } \\
\hline 8 & 200 & 15 & $0.9648(.0648)$ & $.2201(.0471)$ & $.6317(.1373)$ & $.3903(.0894)$ & 25.79 \\
\hline 15 & 200 & 15 & $0.9647(.0476)$ & $.2172(.0296)$ & $.6718(.0787)$ & $.3679(.0516)$ & 53.77 \\
\hline 30 & 200 & 15 & $0.9503(.0369)$ & $.2197(.0257)$ & $.6946(.0735)$ & $.3370(.0306)$ & 136.88 \\
\hline 50 & 200 & 15 & $0.9469(.0286)$ & $.2344(.0221)$ & $.7079(.0700)$ & $.3091(.0230)$ & 263.49 \\
\hline 8 & 200 & 50 & $0.9891(.0671)$ & $.2111(.0470)$ & $.6869(.1069)$ & $.3843(.0773)$ & 85.07 \\
\hline 15 & 200 & 50 & $0.9871(.0482)$ & $.2071(.0306)$ & $.6945(.0721)$ & $.3850(.0523)$ & 182.87 \\
\hline 30 & 200 & 50 & $0.9773(.0384)$ & $.2056(.0256)$ & $.7066(.0632)$ & $.3727(.0316)$ & 423.29 \\
\hline \multirow[t]{2}{*}{50} & 200 & 50 & $0.9734(.0289)$ & $.2177(.0222)$ & $.7056(.0605)$ & $.3508(.0236)$ & 837.40 \\
\hline & & & \multicolumn{5}{|c|}{ Bias adjusted SML } \\
\hline 8 & 200 & 15 & $0.9884(.0675)$ & $.2089(.0488)$ & $.6917(.1327)$ & $.3876(.0936)$ & 26.01 \\
\hline 15 & 200 & 15 & $0.9818(.0490)$ & $.2097(.0306)$ & $.6993(.0790)$ & $.3805(.0537)$ & 54.20 \\
\hline 30 & 200 & 15 & $0.9628(.0377)$ & $.2143(.0267)$ & $.7102(.0757)$ & $.3535(.0318)$ & 137.75 \\
\hline 50 & 200 & 15 & $0.9565(.0293)$ & $.2313(.0228)$ & $.7174(.0720)$ & $.3230(.0237)$ & 264.98 \\
\hline 8 & 200 & 50 & $0.9987(.0682)$ & $.2064(.0477)$ & $.7058(.1048)$ & $.3845(.0788)$ & 85.80 \\
\hline 15 & 200 & 50 & $0.9958(.0491)$ & $.2028(.0312)$ & $.7036(.0723)$ & $.3946(.0536)$ & 184.42 \\
\hline 30 & 200 & 50 & $0.9864(.0393)$ & $.1996(.0266)$ & $.7150(.0649)$ & $.3885(.0331)$ & 426.18 \\
\hline 50 & 200 & 50 & $0.9820(.0296)$ & .2116 (.0234) & $.7135(.0629)$ & $.3677(.0247)$ & 842.32 \\
\hline
\end{tabular}

SML-Serially correlated disturbances; random component; the Polys mode Root mean squared erro

\begin{tabular}{|ccc|cccc|cccc|}
\hline$T$ & $n$ & $m$ & $\beta$ & $\lambda$ & $\sigma$ & $\rho$ & $\beta$ & $\lambda$ & $\sigma$ & $\rho$ \\
\hline & & & \multicolumn{3}{|c|}{ Bias unadjusted SML } & \multicolumn{5}{|c|}{ Bias adjusted SML } \\
\hline 8 & 200 & 15 & .0736 & .0511 & .1565 & .0898 & .0684 & .0495 & .1334 & .0942 \\
15 & 200 & 15 & .0592 & .0342 & .0861 & .0607 & .0522 & .0321 & .0793 & .0570 \\
30 & 200 & 15 & .0618 & .0323 & .0744 & .0700 & .0529 & .0302 & .0756 & .0563 \\
50 & 200 & 15 & .0603 & .0409 & .0699 & .0938 & .0525 & .0387 & .0726 & .0805 \\
& & & & & & & & & & \\
8 & 200 & 50 & .0679 & .0482 & .1086 & .0787 & .0681 & .0480 & .1046 & .0802 \\
15 & 200 & 50 & .0498 & .0313 & .0730 & .0543 & .0492 & .0313 & .0723 & .0538 \\
30 & 200 & 50 & .0445 & .0262 & .0631 & .0417 & .0415 & .0260 & .0653 & .0350 \\
50 & 200 & 50 & .0392 & .0283 & .0604 & .0546 & .0346 & .0261 & .0631 & .0407 \\
& & & & & & & & & & \\
\hline
\end{tabular}




\section{Renewal Models}

6.1 Renewal Model with Serially Correlated Disturbances

A renewal model with a discount factor $\delta$ is considered. This model for our study is specified as

$$
y_{i t}^{*}=x_{i t} \beta+\lambda \sum_{j=1}^{b} \delta^{j-1} \prod_{i=1}^{j} d_{i, \ell-1}+\epsilon_{i t}
$$

where

$$
\epsilon_{i t}=\rho \epsilon_{i, t-1}+w_{i t}
$$

and $w_{i t}$ are i.i.d. $N(0,1)$. The process is assumed to start at the initial period with $c_{i 0}=0$. The renewal model captures the essence that the current continuous duration in one state is a determinant of the decision to remain in or exit the state. The simulated log likelihood function for this model is

$$
\mathcal{L}=\sum_{i=1}^{n} \ln \left\{\frac{1}{m} \sum_{j=1}^{m} \prod_{t=1}^{T} \Phi\left[D_{i t}\left(\beta x_{i t}+\lambda \sum_{j=1}^{t} \delta^{j-1} \prod_{l=1}^{j} d_{i, t-1}+\rho \epsilon_{i, l-1}^{(j)}\right)\right]\right\} .
$$

The discount factor $\delta$ is assumed to be a known constant with the value of 0.7 . The true parameters are set to $\beta=1, \lambda=0.2$ and $\rho=0.4$ as for the other models.

The SMLE and bias-corrected estimates for this model and their root mean square errors are reported in Tables 9.A and 9.B. The SMLE for this model are quite good. There is some biases in estimates of $\rho$. These biases can be reduced by increasing $m$ to 50 or by the bias correction procedure. These estimates are good for all the panels with $T$ up to 50 . Estimates of both the dynamic effect $\lambda$ and the serial correlation coefficient $\rho$ of this model are better in terms of smaller biases and standard errors than estimates of the Markov model in Table 4. Comparing them with estimates of the Polya model in Table 9, these estimates are only slightly worse. These results are compatible with model specification in that the true state dependence in the Renewal model is stronger than the state dependence in the Markov model but is weaker than the state dependence of the Polya model.

6.2 Renewal Modele with Unobserved Heterogeneity and Serially Correlated Disturbances Incorporating a random component $\xi$, the model with a discount factor $\delta$ is

$$
y_{i t}^{*}=x_{i t} \beta+\lambda \sum_{j=1}^{t} \delta^{j-1} \prod_{i=1}^{j} d_{i, t-1}+\sigma \xi_{i}+\epsilon_{i t} \text {, }
$$

where

$$
c_{i t}=\rho \epsilon_{i, t-1}+w_{i t}
$$

and $\xi_{i}$ and $w_{i t}$ are i.i.d. $N(0,1)$. The process is asesumed to start at the initial period with $\epsilon_{i 0}=0$. The simulated log likelihood function for this model is

$$
\mathcal{L}=\sum_{i=1}^{n} \ln \left\{\frac{1}{m} \sum_{j=1}^{m} \prod_{t=1}^{T} \Phi\left[D_{i t}\left(\beta x_{i t}+\lambda \sum_{j=1}^{t} \delta^{j-1} \prod_{l=1}^{j} d_{i, t-1}+\sigma \xi_{i}^{(j)}+\rho \epsilon_{i, t-1}^{(j)}\right)\right]\right\} .
$$

The discount factor is known to be 0.7 . The sample dats are generated with $\beta=1, \lambda=0.2, \sigma^{2}=0.5$, and $\rho=0.4$.

Results for the estimation of this model are reported on Tables 10.A and 10.B. There are upward biases in the SMLE of $\lambda$ and downward biases in estimates of $\rho$. The magnitude of bias is increasing as $T$ becomes longer. Biases in estimates of $\beta$ and $\rho$ are relatively small. With large $m=50$, all the biases are reduced. The bias-correction procedure has also reduced the bias. The bias-corrected estimates with $m=50$ are pretty good. They have small biases and small root mean equare errors. The model can be cetimated adequately for panels with $\boldsymbol{T}$ up to 50 . As the state dependence in this model is relatively weaker than a corresponding Polya model, estimates of this model are slightly less accurate in terms of biases and atandard errors than estimates of the Polys model in Table 8. On the other hand, these estimates are much better than estimates of the Markov model with unknown heterogeneity in Table 6. The computing time coet of this model with unobserved heterogeneity is slightly lese than the time cost of the renewal model without unobserved heterogeneity in (6.1). It is about the same computing cost for a Polys model with unobeerved heterogeneity. 
Table 9.A

SML-oerial correlated disturbances; the Renewal model True parameters: $\beta=1, \lambda=0.2$, and $\rho=0.4$

\begin{tabular}{|ccc|cccc|}
\hline$T$ & $n$ & $m$ & $\beta$ & $\lambda$ & $\rho$ & time \\
\hline & & & \multicolumn{4}{|c|}{ Bias unadjusted SML } \\
\hline \\
8 & 200 & 15 & $0.9953(.0559)$ & $.2077(.0392)$ & $.3757(.0611)$ & 27.90 \\
15 & 200 & 15 & $0.9871(.0432)$ & $.2104(.0259)$ & $.3716(.0363)$ & 65.01 \\
30 & 200 & 15 & $0.9806(.0316)$ & $.2117(.0161)$ & $.3613(.0252)$ & 142.37 \\
50 & 200 & 15 & $0.9755(.0240)$ & $.2131(.0106)$ & $.3476(.0188)$ & 262.83 \\
& & & & & & \\
8 & 200 & 50 & $1.0040(.0561)$ & $.2004(.0402)$ & $.3969(.0628)$ & 95.30 \\
15 & 200 & 50 & $0.9970(.0442)$ & $.2037(.0272)$ & $.3936(.0381)$ & 221.65 \\
30 & 200 & 50 & $0.9946(.0328)$ & $.2039(.0171)$ & $.3905(.0270)$ & 473.70 \\
50 & 200 & 50 & $0.9909(.0244)$ & $.2048(.0113)$ & $.3799(.0207)$ & 868.00 \\
& & & & & & \\
\hline & & & & Bias adjusted & SML & \\
\hline \multirow{2}{*}{} & & & & & & \\
8 & 200 & 15 & $1.0054(.0575)$ & $.1996(.0402)$ & $.3997(.0636)$ & 28.13 \\
15 & 200 & 15 & $0.9982(.0444)$ & $.2032(.0269)$ & $.3968(.0382)$ & 65.42 \\
30 & 200 & 15 & $0.9919(.0326)$ & $.2051(.0168)$ & $.3860(.0267)$ & 143.21 \\
50 & 200 & 15 & $0.9856(.0245)$ & $.2075(.0109)$ & $.3696(.0196)$ & 264.27 \\
& & & & & & \\
8 & 200 & 50 & $1.0079(.0567)$ & $.1972(.0407)$ & $.4059(.0638)$ & 96.02 \\
15 & 200 & 50 & $1.0018(.0449)$ & $.2004(.0277)$ & $.4043(.0393)$ & 223.03 \\
30 & 200 & 50 & $1.0011(.0336)$ & $.1999(.0177)$ & $.4041(.0283)$ & 476.66 \\
50 & 200 & 50 & $0.9981(.0249)$ & $.2007(.0117)$ & $.3950(.0219)$ & 872.76 \\
& & & & & \\
\hline
\end{tabular}

Table 9.B

SML-Serially correlated disturbances; the Renewal model Root mean squared errors

\begin{tabular}{|ccc|ccc|ccc|}
\hline$T$ & $n$ & $m$ & $\beta$ & $\lambda$ & $\rho$ & $\beta$ & $\lambda$ & $\rho$ \\
\hline & & & \multicolumn{2}{|c|}{ Bias unadjusted SML } & \multicolumn{3}{|c|}{ Bias adjusted SML } \\
\hline 8 & 200 & 15 & .0560 & .0398 & .0656 & .0577 & .0401 & .0635 \\
15 & 200 & 15 & .0450 & .0279 & .0461 & .0443 & .0270 & .0383 \\
30 & 200 & 15 & .0371 & .0199 & .0461 & .0335 & .0175 & .0301 \\
50 & 200 & 15 & .0343 & .0168 & .0557 & .0284 & .0132 & .0362 \\
& & & & & & & & \\
8 & 200 & 50 & .0561 & .0402 & .0628 & .0571 & .0407 & .0640 \\
15 & 200 & 50 & .0442 & .0274 & .0386 & .0448 & .0277 & .0394 \\
30 & 200 & 50 & .0332 & .0175 & .0286 & .0336 & .0177 & .0286 \\
50 & 200 & 50 & .0260 & .0123 & .0288 & .0249 & .0117 & .0224 \\
\end{tabular}

Table 10.A

SML-Serially correlated disturbances; random component; the Renewal model True parameters: $\beta=1, \lambda=0.2, \sigma=\sqrt{0.5}$, and $\rho=0.4$

\begin{tabular}{|c|c|c|c|c|c|c|c|}
\hline $\bar{T}$ & $n$ & $m$ & $\bar{\beta}$ & $\lambda$ & $\sigma$ & $\boldsymbol{\rho}$ & time \\
\hline & & & \multicolumn{5}{|c|}{ Bies unadjusted SML } \\
\hline 8 & 200 & 15 & $0.9645(.0646)$ & $.2249(.0558)$ & $.6342(.1251)$ & $.3815(.0943)$ & 27.79 \\
\hline 15 & 200 & 15 & $0.9610(.0464)$ & $.2318(.0343)$ & $.6617(.0783)$ & $.3458(.0587)$ & 55.96 \\
\hline 30 & 200 & 15 & $0.9507(.0355)$ & $.2367(.0269)$ & $.6796(.0635)$ & $.3220(.0376)$ & 131.49 \\
\hline 50 & 200 & 15 & $0.9465(.0288)$ & $.2562(.0223)$ & .7008 (.0699) & $.2876(.0259)$ & 260.57 \\
\hline 8 & 200 & 50 & $0.9894(.0670)$ & $.2146(.0554)$ & $.6873(.1056)$ & $.3806(.0884)$ & 93.14 \\
\hline 15 & 200 & 50 & $0.9862(.0464)$ & $.2135(.0366)$ & $.6933(.0755)$ & $.3746(.0583)$ & 202.58 \\
\hline 30 & 200 & 50 & $0.9736(.0354)$ & $.2191(.0265)$ & $.6746(.0555)$ & $.3607(.0370)$ & 440.09 \\
\hline \multirow[t]{2}{*}{50} & 200 & 50 & $0.9757(.0292)$ & $.2285(.0222)$ & $.6991(.0488)$ & $.3444(.0265)$ & 820.62 \\
\hline & & & \multicolumn{5}{|c|}{ Bias adjusted SML } \\
\hline 8 & 200 & 15 & $0.9882(.0671)$ & $.2120(.0577)$ & $.6925(.1211)$ & $.3832(.0981)$ & 28.00 \\
\hline 15 & 200 & 15 & $0.9788(.0476)$ & $.2201(.0357)$ & $.6898(.0788)$ & $.3635(.0613)$ & 56.39 \\
\hline 30 & 200 & 15 & $0.9641(.0362)$ & $.2261(.0279)$ & $.6968(.0650)$ & $.3437(.0390)$ & 132.36 \\
\hline 50 & 200 & 15 & $0.9569(.0296)$ & $.2489(.0231)$ & $.7120(.0717)$ & $.3059(.0268)$ & 262.02 \\
\hline 8 & 200 & 50 & $0.9989(.0680)$ & $.2091(.0562)$ & .7054 (.1035) & $.3833(.0904)$ & 93.88 \\
\hline 15 & 200 & 50 & $0.9954(.0473)$ & $.2061(.0377)$ & $.7036(.0760)$ & $.3880(.0601)$ & 204.02 \\
\hline 30 & 200 & 50 & $0.9833(.0359)$ & $.2089(.0277)$ & $.6839(.0568)$ & $.3813(.0387)$ & 443.03 \\
\hline 50 & 200 & 50 & $0.9850(.0299)$ & $.2169(.0234)$ & $.7098(.0500)$ & $.3679(.0278)$ & 825.54 \\
\hline
\end{tabular}

Table 10.B

SML-Serially correlated disturbances; random component; the Renewal model Root mean squared error

\begin{tabular}{|ccc|cccc|cccc|}
\hline$T$ & $n$ & $m$ & $\beta$ & $\lambda$ & $\sigma$ & $\rho$ & $\beta$ & $\lambda$ & $\sigma$ & $\rho$ \\
\hline & & & \multicolumn{3}{|c|}{ Bias unadjusted SML } & \multicolumn{3}{|c|}{ Bias adjusted SML } \\
\hline \multirow{2}{*}{} & 200 & 15 & .0736 & .0610 & .1446 & .0959 & .0681 & .0588 & .1217 & .0994 \\
15 & 200 & 15 & .0605 & .0467 & .0904 & .0799 & .0520 & .0409 & .0806 & .0713 \\
30 & 200 & 15 & .0607 & .0454 & .0691 & .0866 & .0509 & .0382 & .0657 & .0685 \\
50 & 200 & 15 & .0607 & .0605 & .0701 & .1153 & .0523 & .0540 & .0717 & .0978 \\
& & & & & & & & & & \\
8 & 200 & 50 & .0677 & .0572 & .1073 & .0904 & .0679 & .0569 & .1033 & .0918 \\
15 & 200 & 50 & .0483 & .0389 & .0766 & .0635 & .0474 & .0381 & .0759 & .0612 \\
30 & 200 & 50 & .0441 & .0326 & .0642 & .0539 & .0396 & .0291 & .0612 & .0429 \\
50 & 200 & 50 & .0380 & .0361 & .0494 & .0616 & .0335 & .0289 & .0500 & .0424 \\
& & & & & & & & & & \\
\hline
\end{tabular}




\section{Habit Persistent Modela}

7.1 Models with Serially Correlated Disturbances

The habit persistent model is apecified as follows:

$$
y_{i t}^{i}=x_{i t} \beta+\lambda y_{i, t-1}^{i}+\epsilon_{i t},
$$

where

$$
\epsilon_{i t}=\rho \epsilon_{i, t-1}+w_{i t}
$$

and $w_{i t}$ are i.i.d. $N(0,1)$. The process starts at the initial period with $y_{i 0}^{*}=0$ and $c_{i 0}=0$. The simulated log likelihood function for this model is

$$
\mathcal{L}=\sum_{i=1}^{n} \ln \left\{\frac{1}{m} \sum_{j=1}^{m} \prod_{t=1}^{T} \Phi\left[D_{i t}\left(\beta x_{i t}+\lambda y_{i, t-1}^{(j)}+\rho \epsilon_{i, t-1}^{(j)}\right)\right]\right\} .
$$

The true parameters are $\beta=1, \lambda=0.2$, and $\rho=0.4$.

The SMLE and bias-corrected estimates and their root mean square errors are reported in Tables 11.A and 11.B. There are downward biases in the SMLE for all the parameters $\beta, \lambda$ and $\rho$. Biases for $\beta$ and $\lambda$ are relatively amaller than biases for $\rho$. With $m$ increased to 50 , biases are reduced and those biases for $\beta$ and $\lambda$ are omall. The bias-correction procedure can further reduce bias and biases for $\rho$ can become small. Bias-corrected estimates have also smaller mean squared errors than those of the unadjusted SMLE. The parameters in this model can be accurately estimated with $\boldsymbol{T}$ up to 30 or even 50 . Comparing these eatimateo with estimates of the previous dynamic models, the main difference is on the direction of bias of estimates of the dynamic effect $\lambda$. While estimates of $\lambda$ tend to be upward in Markov, Polya and Renewal models, the bias in the habit persistent model tends to be downward. The dynamic coefficient $\lambda$ can be better estimated in terms of smaller bias and standard error than the corresponding estimates of the Markov model in Table 4. However, they are not better estimated than the ones in Polya and Renewal models. The habit persistent model above is a first order latent Markov model. One may suspect that the dynamic effect for this model is atronger than the dynamic effect of the corresponding Markov model but is weaker than the accumulated effects of Polya and Renewal models. On the other hand, estimates of $\beta$ and $\rho$ in this model have larger root mean equare errors than the ones in the Markov model. The $\beta$ and $\rho$ in Polya and Renewal modelo are also better estimated. In terms of time coot, it takes more time to estimate the habit persistence model than the Markoy model but it costa lese than the computation of Polya and Renewal modelo.
7.2 Models with Unobserved Heterogeneity and Serially Correlated Disturbances

The habit persistent model with a random component is specified as follows:

$$
y_{i t}^{*}=x_{i t} \beta+\lambda y_{i, 1-1}^{*}+\sigma \xi_{i}+c_{i t}
$$

where

$$
\epsilon_{i t}=\rho \epsilon_{i, t-1}+w_{i t}
$$

and $\xi_{i}$ and $w_{i t}$ are i.i.d. $N(0,1)$. The proces is assumed to start at the initial period with $y_{i 0}^{*}=0$ and $\epsilon_{i 0}=0$. The simulated log likelihood function for this model is

$$
\mathcal{L}=\sum_{i=1}^{n} \ln \left\{\frac{1}{m} \sum_{j=1}^{m} \prod_{t=1}^{T} \Phi\left[D_{i t}\left(\beta x_{i t}+\lambda y_{i, t-1}^{(j)}+\sigma \xi_{i}^{(j)}+\rho \epsilon_{i, t-1}^{(j)}\right)\right]\right\} .
$$

As for the other models with unknown heterogeneity, the true parameters are $\beta=1, \lambda=0.2, \sigma^{2}=0.5$, and $\rho=0.4$ to generate sample observations.

Tables 12.A and 12.B report results for this model. With the additional heterogeneity, estimates of $\beta, \lambda$ and $\rho$ have larger downward biases than the ones for the habit persistent model without unobeerved heterogeneity. The magnitude of bise becomes larger as $T$ becomes longer. For $\sigma$, there is some bias in its estimate for panels with small $T$. As $m$ increases, biases decrease. The bias-corrected procedure can further reduce bias and root mean squares errors are in general also smaller. With $m=50$ and the bias-corrected procedure, estimates are reasonably good for panels with $T$ up to 15 or 30 . Estimates of $\lambda$ and $\rho$ are more accurate than corresponding estimates of the Markov model with unknown heterogeneity in Table $\theta$ in terms of bias and root mean square error. On the other hand, estimates of $\beta$ and $\rho$ in this model are lese precise in terms of bias and root mean square error than estimates of the Markov model with unobserved heterogeneity. Estimates of this habit persistent model are lese accurate than estimates of the corresponding Polya and Renewal modele. 
Table 11.A

SML arial correlation; the Habit Pemistence mode

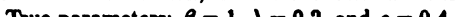

\begin{tabular}{|ccc|cccc|}
\hline$T$ & $n$ & $m$ & $\beta$ & $\lambda$ & $\rho$ & time \\
\hline & & & \multicolumn{4}{|c|}{ Bies unadjusted SML } \\
\hline 8 & 200 & 15 & $0.9849(.0577)$ & $.1932(.0521)$ & $.3666(.0660)$ & 26.04 \\
15 & 200 & 15 & $0.9737(.0450)$ & $.1960(.0358)$ & $.3512(.0453)$ & 56.81 \\
30 & 200 & 15 & $0.9644(.0303)$ & $.1887(.0267)$ & $.3365(.0339)$ & 133.45 \\
50 & 200 & 15 & $0.9546(.0255)$ & $.1863(.0224)$ & $.3172(.0287)$ & 247.35 \\
& & & & & & \\
8 & 200 & 50 & $0.9952(.0591)$ & $.1977(.0511)$ & $.3852(.0646)$ & 87.70 \\
15 & 200 & 50 & $0.9886(.0463)$ & $.2013(.0342)$ & $.3776(.0422)$ & 194.82 \\
30 & 200 & 50 & $0.9823(.0315)$ & $.1942(.0249)$ & $.3699(.0313)$ & 441.81 \\
50 & 200 & 50 & $0.9734(.0258)$ & $.1902(.0209)$ & $.3536(.0258)$ & 811.17 \\
& & & & & & \\
\hline & & & & Bias adjusted SML & \\
\hline \multirow{2}{*}{8} & 200 & 15 & $0.9973(.0593)$ & $.1976(.0515)$ & $.3892(.0647)$ & 26.25 \\
15 & 200 & 15 & $0.9866(.0464)$ & $.1992(.0349)$ & $.3753(.0444)$ & 57.22 \\
30 & 200 & 15 & $0.9754(.0310)$ & $.1908(.0257)$ & $.3576(.0326)$ & 134.35 \\
50 & 200 & 15 & $0.9636(.0257)$ & $.1874(.0222)$ & $.3343(.0277)$ & 248.78 \\
& & & & & & \\
8 & 200 & 50 & $1.0003(.0599)$ & $.1998(.0509)$ & $.3939(.0642)$ & 88.42 \\
15 & 200 & 50 & $.9955(.0472)$ & $.2036(.0338)$ & $.3892(.0420)$ & 196.21 \\
30 & 200 & 50 & $.9905(.0321)$ & $.1967(.0244)$ & $.3843(.0307)$ & 444.69 \\
50 & 200 & 50 & $.9812(.0261)$ & $.1920(.0207)$ & $.3676(.0253)$ & 816.01 \\
& & & & & & \\
\hline
\end{tabular}

Table 11.B

SML-Serially correlation; the Habit Persistence model

Root mean squared errors

\begin{tabular}{|ccc|ccc|ccc|}
\hline$T$ & $n$ & $m$ & $\beta$ & $\lambda$ & $\rho$ & $\beta$ & $\lambda$ & $\rho$ \\
\hline & & & \multicolumn{2}{|c|}{ Bias unadjusted SML } & \multicolumn{3}{|c|}{ Bias adjusted SML } \\
\hline $\mathbf{8}$ & 200 & 15 & .0595 & .0525 & .0738 & .0593 & .0515 & .0655 \\
15 & 200 & 15 & .0521 & .0359 & .0665 & .0482 & .0349 & .0507 \\
30 & 200 & 15 & .0467 & .0289 & .0720 & .0395 & .0273 & .0534 \\
50 & 200 & 15 & .0520 & .0262 & .0876 & .0445 & .0255 & .0713 \\
& & & & & & & & \\
8 & 200 & 50 & .0592 & .0511 & .0661 & .0598 & .0508 & .0644 \\
15 & 200 & 50 & .0476 & .0342 & .0477 & .0473 & .0340 & .0433 \\
30 & 200 & 50 & .0361 & .0256 & .0434 & .0335 & .0246 & .0344 \\
50 & 200 & 50 & .0370 & .0231 & .0531 & .0321 & .0222 & .0411 \\
\end{tabular}

Table 12.A

SML-Serially correlation; random True parameters: $\beta=1, \lambda=0.2, \sigma=\sqrt{0.5}$, and $\rho=0.4$

\begin{tabular}{|c|c|c|c|c|c|c|c|}
\hline $\boldsymbol{T}$ & $n$ & $m$ & $\boldsymbol{\beta}$ & $\boldsymbol{\lambda}$ & $\sigma$ & p & time \\
\hline & & & \multicolumn{5}{|c|}{ Bias unadjusted SML } \\
\hline 8 & 200 & 15 & $0.9560(.0692)$ & $.1833(.0747)$ & $.6292(.1511)$ & $.4027(.1366)$ & 33.62 \\
\hline 15 & 200 & 15 & $0.9446(.0467)$ & $.1816(.0602)$ & $.6836(.0899)$ & $.3560(.0928)$ & 73.32 \\
\hline 30 & 200 & 15 & $0.9134(.0356)$ & $.1772(.0450)$ & $.6978(.0818)$ & $.3127(.0569)$ & 164.78 \\
\hline 50 & 200 & 15 & $0.9035(.0294)$ & $.1683(.0395)$ & $.7090(.0790)$ & $.2922(.0482)$ & 289.60 \\
\hline 8 & 200 & 50 & $0.9872(.0711)$ & $.1992(.0651)$ & $.6893(.1104)$ & $.3842(.1086)$ & 109.89 \\
\hline 15 & 200 & 50 & $0.9771(.0497)$ & $.1977(.0500)$ & $.7013(.0809)$ & $.3716(.0740)$ & 241.21 \\
\hline 30 & 200 & 50 & $0.9489(.0367)$ & $.1929(.0398)$ & $.6962(.0788)$ & $.3458(.0486)$ & 560.23 \\
\hline \multirow[t]{2}{*}{50} & 200 & 50 & $0.9402(.0294)$ & $.1818(.0301)$ & $.6958(.0671)$ & $.3298(.0356)$ & 1013.38 \\
\hline & & & \multicolumn{5}{|c|}{ Bias adjusted SML } \\
\hline 8 & 200 & 15 & $0.9789(.0727)$ & $.1918(.0700)$ & $.6740(.1465)$ & $.4004(.1333)$ & 33.85 \\
\hline 15 & 200 & 15 & $0.9623(.0482)$ & $.1872(.0563)$ & $.7030(.0903)$ & $.3680(.0880)$ & 73.76 \\
\hline 30 & 200 & 15 & $0.9251(.0366)$ & $.1791(.0427)$ & $.7087(.0830)$ & .3265 (.0542) & 165.70 \\
\hline 50 & 200 & 15 & $0.9114(.0303)$ & $.1688(.0385)$ & $.7157(.0800)$ & $.3028(.0466)$ & 291.07 \\
\hline 8 & 200 & 50 & $0.9984(.0727)$ & $.2035(.0635)$ & $.7021(.1073)$ & $.3857(.1061)$ & 110.64 \\
\hline 15 & 200 & 50 & $0.9894(.0508)$ & $.2024(.0483)$ & .7068 (.0812) & $.3828(.0720)$ & 242.70 \\
\hline 30 & 200 & 50 & $0.9610(.0378)$ & $.1967(.0383)$ & $.7019(.0801)$ & $.3603(.0469)$ & 563.22 \\
\hline 50 & 200 & 50 & $0.9497(.0300)$ & $.1839(.0294)$ & $.7016(.0686)$ & $.3430(.0345)$ & 1018.62 \\
\hline
\end{tabular}

Table 12.B

SML-Serially correlation; random component; the Habit Peraistence model Root mean squared error

\begin{tabular}{|c|c|c|c|c|c|c|c|c|c|c|}
\hline \multirow[t]{2}{*}{$T$} & $n$ & $m$ & $\beta$ & $\lambda$ & $\sigma$ & $\bar{p}$ & $\bar{\beta}$ & $\begin{array}{ll}\lambda \\
\lambda\end{array}$ & $\sigma$ & $p$ \\
\hline & & & \multicolumn{4}{|c|}{ Bias unadjusted SML } & \multicolumn{4}{|c|}{ Bian adjusted SML } \\
\hline 8 & 200 & 15 & .0819 & .0764 & .1698 & .1364 & .0755 & .0703 & .1500 & .1330 \\
\hline 15 & 200 & 15 & .0725 & .0629 & .0928 & .1026 & .0611 & .0577 & .0902 & .0935 \\
\hline 30 & 200 & 15 & .0936 & .0504 & .0822 & .1042 & .0834 & .0475 & .0829 & .0913 \\
\hline 50 & 200 & 15 & 1009 & .0505 & .0789 & .1181 & 0937 & .0495 & .0803 & .1078 \\
\hline 8 & 200 & 50 & .0721 & .0650 & .1117 & .1096 & .0726 & .0634 & .1072 & .1068 \\
\hline 15 & 200 & 50 & .0547 & .0499 & .0809 & .0791 & .0518 & .0483 & .0810 & .0738 \\
\hline 30 & 200 & 50 & .0628 & .0404 & .0794 & .0728 & .0543 & .0384 & .0801 & .0614 \\
\hline 50 & 200 & 50 & .0666 & .0352 & .0679 & .0787 & .0585 & .0335 & .0687 & .0666 \\
\hline
\end{tabular}




\section{Conclusions}

This article has reported Monte Carlo results of simulated maximum likelihood methods for the estimation of discrete choice regression and dynamic models. Choice probabilities are simulated with the GHK recursive estimator. We investigate finite sample performance of the simulated likelihood estimator and a bias-correction procedure which can eliminate the leading bias due to aimulation. The diacrete choice models are panel choice models introduced in Heckman (1981a), that include discrete choice regression models with true state dependence, spurious correlation, and unobserved heterogeneity. Even with a binary choice in each time period, the pattern or path of choices can be very large as time periods become longer. Our intention is two folds. We would like to investigate finite sample performance of the simulated maximum likelihood estimator for panel data dynamic models, limitation of simulated likelihood methods, and the value of long panels compared with relatively short panels. The second purpose is to study the econometric estimation of various interesting dynamic discrete choice models, the ability of estimating dynamic effects, and the distinction of true state dependence from spurious state dependence and unobserved heterogeneity.

As an cotimation method, the simulated maximum likelihood approach is computationally tractable and numerically stable. We have experimented with panel data with time length up to 100. There is not a single case of failure of convergence of the iterative maximization algorithm. Modelo with moderate serial correlation can be more accurately estimated than models with high serial correlation in terms of smaller bias and standard error. Regression models are better estimated than Markov state dependence models. Dynamic models with strong dynamic effects such as the generalized Polya model and the renewal model can be better estimated than the Markov model and the latent Markov model. In terms of time coot, the eatimation of generalized Polya and renewal modele are more expensive than the estimation of the Markov model and the latent Markov model. With a small number of simulation draws for the construction of simulator and relatively long panels, dynamic effects of Markov, Polya and renewal models can be overestimated and the spurious correlation can be underestimated. Only for the latent Markov model, both the dynamic and spurious correlation effects are underestimated. While standard errors of eatimates tend to be amaller for panels with longer periods, the bias for panel data with long periods such as 100 can dominate the standard error and results in a larger root mean square error in some of the modelo. Biases of the SMLE can usually be reduced with a larger number of simulated variables for the construction of simulator and by the bias correction procedure. With the bias correction procedure and a moderate number of aimulated draws for the simulator, say 50, most of the dynamic models with moderate sutocorrelation can be adequately eatimated for panels up to 30 periods. Due to strong state dependence, Polya and Renewal modelo can be adequately estimated up to 50 periodo in our Monte Carlo experiments.

While our Monte Carlo experiments have studied many important aspects of simulation eatimation methods and efficient estimation of dynamic panel statistical models, many interesting isesues have not been addressed. We have not investigated statistical inference procedures on model discrimination or model 10 lection based on simulation estimation methoda. For the estimation of dynamic panel models, we have not investigated the initial value problem, which can be quite difficult and is an open challenging question (Heckman (1981b)]. Furthermore, we have not investigated intertemporal optimisation dynamic choice modelo where extra complexity will be encountered in backward evaluation of value functions in the presence of serially correlated disturbances [Pakes (1987), Rust (1987), and Wolpin (1984)]. All these issues remain for further investigation. 


\section{Reference:}

Borech-Supan, A. and V.A. Hajivasoiliou (1993). "Smooth Unbiased Multivariate Probability Simulatore for Maximum Likelihood Estimation of Limited Dependent Variable Models." Journal of Econometrica 58: $347-368$.

Butler, J.S. and R. Moffitt (1982). "A Computationally Efficient Quadrature Procedure for the One-Factor Multinomial Probit Model." Econometrice 50: 761-764.

Chamberlain, G. (1982). "Multivariate Regression Models for Panel Data." Journal of Econometrics 18: 5-46.

Geweke, J. (1989). "Efficient Simulation from the Multivariate Normal Distribution Subject to Linear Inequality Constraints and the Evaluation of Constraint Probabilities." memo. Duke University, Durham, NC.

Gourieroux, C. and A. Monfort (1993). "Simulation-based Inference: A Survey with Special Reference to Panel Data Models." Journal of Econometrics 59: 5-33.

Hajivassiliou, V. and D. McFadden (1990). "The Method of Simulated Seores for the Eatimation of LDV modelo: With an Application to the Problem of External Debt Crises." Cowles Foundation Discuseion Paper No. 967, Yale University, New Haven, CT.

Heckman, J.J. (1978). "Simple Statistical Models for Discrete Panel Data Developed and Applied to Test the Hypothesis of True State Dependence Against the Hypothesis of Spurious State Dependence." Annalea de l'INSEE 30-1: 227-269.

- (1981a). "Statistical Models for Discrete Panel Data," in Structural Analysis of Discrete Data with Econometric Applications, ed. by C.F. Manski and D. McFadden, pp.114-178. Cambridge: MIT Prese.

- (1981b). "The Incidental Parameters Problem and the Problem of Initial Conditions in Eatimating - Discrete Time-Discrete Data Stochastic Processo." In Structural Analysis of Discrete Date with Econometric Applications, ed. by C.F. Manski and D. McFadden, pp. 179-195. Cambridge: MIT Preen.

- (1981c). "Heterogeneity and State Dependence." In Studies in Labor Markets, ed. by S. Roeen, pp. 91-139. Chicago: University of Chicago Press.

Haiso, C. (1986). Analygis of Panel Date. Cambridge: Cambridge University Prese.
Keane, M.P. (1994). "A Computationally Practical Simulation Eatimator for Panel Data." Econometrice 62: 95-116.

Lee, L.F. (1987). "Non-Parametric Testing of Discrete Panel Data Modelo." Journal of Econometrics 34: 147-177.

Lee, L.F. (1992). "On Efficiency of Methods of Simulated Moments and Maximum Simulated Likelihood Eotimation of Discrete Reaponse Modele." Econometric Theory 8: 518-552.

Lee, L.F. (1993). "Asymptotic Bise in Maximum Simulated Likelihood Estimation of Discrete Choice Modelo." CREST and Department of Economics Working Paper Series, Number 93-03. The Univenity of Michigan, Ann Arbor, Michigan.

Lerman, S. and C. Manski (1981). "On the Use of Simulated Frequencies to Approximate Choice Probabilities." In Structural Analysis of Discrete Date with Econometric Applications, ed. by C. Maneki and D. McFadden. Cambridge: MIT Prese.

McFadden, D. (1973). "Conditional Logit Analysis of Qualitative Choice Behavior." In Frontiers in Econometrics, ed. P. Zarembka. New York: Academic Press.

McFadden, D. (1989). "A Method of Simulated Moments of Estimation of Discrete Choice Modele without Numerical Integration." Econometrice 57: 995-1026.

Pakes, A. (1987). "Patents as Options: Some Eatimates of the Value of Holding European Patent Stocks." Econometrica 54: 755-784.

Pakes, A. and D. Pollard (1989). "Simulation and the Asymptotics of Optimization Estimators." Econometrica 54: 755-785.

Press, W.B., B.P. Flannery, S.A. Teukolsky, and W.T. Vetterling (1986). Numerical Recipes. New York: Cambridge University Press.

Rust, J. (1987). "Optimal Replacement of GMC Bus Engines: An Empirical Model of Harold Zurcher." Econometrice 55: 999-1035.

Wolpin, K.I. (1984). "An Estimable Dynamic Stochastic Model of Fertility and Child Mortality." Joumal of Political Economy 92: 852-874. 



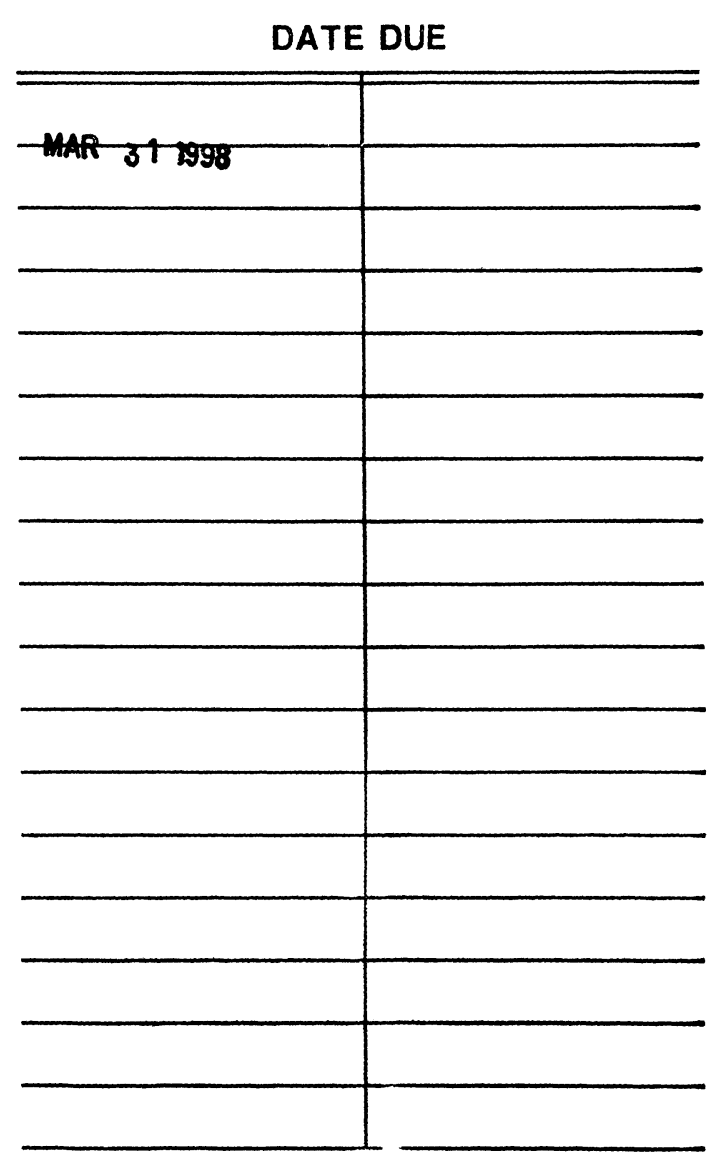

DEMCO, INC. 38-2931 
. 